\title{
Strategic Use of Storage: The Impact of Carbon Policy, Resource Availability, and Technology Efficiency on a Renewable-Thermal Power System
}

\author{
Sébastien Debia \\ Department of Decision Sciences, HEC Montréal \\ Pierre-Olivier Pineau \\ Department of Decision Sciences, HEC Montréal \\ Afzal S. Siddiqui* \\ Department of Statistical Science, University College London, Department of Computer and Systems Sciences, \\ Stockholm University, and Department of Decision Sciences, HEC Montréal
}

\begin{abstract}
Concerns about climate change have spurred governments to reduce carbon emissions by supporting adoption of renewable energy (RE) technologies. Due to the intermittent and location-specific nature of RE technologies, energy storage has become important because it could be used to smooth out temporal disparities in residual demand. Thus, carbon policy has made storage-enabled RE generation more critical to the power sector, and this enhanced position could be exploited by firms to exert market power. Using an equilibrium model, we examine the implications of policy interventions and technological change on the marginal value of energy storage in a power market with RE and thermal generation. In particular, we specify the market conditions under which RE producers with storage strategically shift deployment of their resource to the off-peak period and outline its implications for the marginal value of RE storage. Moreover, we find that even pricetaking RE producers may actually increase off-peak RE production as storage efficiency increases. Consequently, the RE producer's profit decreases with storage efficiency, which conflicts with the social objective of improving storage efficiency. These private and social incentives can be better aligned via a carbon tax, however. Hence, our results may inform the regulatory process governing market design of a power sector with increasing capacities of RE generation and storage.
\end{abstract}

Keywords: Energy storage, market power, carbon policy, renewable energy

\section{Introduction}

In response to the threat of climate change, several jurisdictions have adopted legally binding targets for renewable energy (RE) technologies. Among these are the European Union's objective

${ }^{*}$ Corresponding author

Email address: afzal.siddiqui@ucl.ac.uk (Afzal S. Siddiqui*) 
of obtaining $20 \%$ of energy from RE technologies by the year 2020 with more ambitious targets for 2030 (European Commission, 2009) and California's 33\% target for RE technologies by the year 2020 with a subsequent increase to $50 \%$ by 2030 as part of Senate Bill No. 350 (California Legislative Information, 2015). Consequently, the power sector is undergoing a profound transformation intended to bolster its sustainability by reducing generation from conventional thermal plants that emit $\mathrm{CO}_{2}$ and other greenhouse gases.

While these policy measures have incentivised investment in and operation of RE technologies, the intermittency of wind and solar power has also introduced a need for variation management, e.g., energy storage and transmission capacity. For example, in Germany, the share of RE in the power sector increased to $30 \%$ in 2014 as compared to less than 5\% in 1990 due to policy measures such as feed-in tariffs (von Hirschhausen, 2014). However, absent network reinforcement, the intermittent and location-specific nature of RE technologies could cause congestion management costs to triple in Germany between 2015 and 2020 (Kunz, 2013). Likewise, energy storage has become important because it could be used to smooth out temporal disparities in residual demand. Using stylised equilibrium models, Sioshansi (2010) and Sioshansi (2014) investigate the welfare implications of storage ownership and device efficiency under perfect and imperfect competition. The rationale for the latter investigation is that the enhanced need for storage provides storage operators with the potential to exert additional market power.

A similar perspective has been taken in the literature on strategic hydropower production in a deregulated electricity industry. Focusing on a numerical study of the California power sector, Bushnell (2003) uses an equilibrium approach to illustrate how producers with market power have the incentive to withhold hydropower generation during peak hours and to move it to off-peak hours instead. Thus, the marginal value of water is lower in an industry with market power and may even be negative as posited by Crampes and Moreaux (2001). The welfare implications of greater resource stocks in the presence of market power are also investigated in the resource economics literature (Benchekroun et al., 2010). Indeed, restructuring of the electricity industry has provoked interest in how hydropower producers' incentives to shift generation between peak and off-peak periods are affected (Mathiesen et al., 2011), but a comprehensive analysis of carbon policy, RE resource availability, and storage efficiency on producers' incentives and the value of storage has been lacking. For example, Førsund (2015) develops fundamental modelling principles for hydropower by accounting for pumped hydro storage and intermittency, but his treatment of market power is limited to a single dominant firm. Other works focus on uncertainty in water inflows by modelling the emissions-constrained hydro-thermal scheduling problem but without any strategic analysis (Rebennack et al., 2012). Indeed, a survey of the literature by Steegel et al. (2014) points out that there are relatively few models on hydropower strategic behaviour. This is in contrast to other energy markets, e.g., the natural gas one, in which it is typical to investigate strategic behaviour with storage and interactions with other fuels (Abada et al., 2013; Gabriel et al., 2013). Moreover, recent studies that do investigate strategic equilibria with a conventional-wind-storage system typically do not incorporate the effects of carbon policy or permit analytical solutions (Shahmohammadi et 
al., 2018). In this paper, we address how carbon policy and strategic behaviour in an electricity industry interact in order to formalise the conditions under which RE producers with storage behave strategically and its implications for the marginal value of $\mathrm{RE}$ storage.

We develop a two-period equilibrium model with an RE and a thermal producer. Focusing on interior solutions under both perfect competition and Cournot oligopoly, we first prove analytically that the main result of Bushnell (2003), i.e., more off-peak RE production under Cournot oligopoly vis-à-vis perfect competition, holds without a carbon tax. However, once the carbon tax exceeds a certain threshold, the incentive of the RE producer to generate more in the off-peak period under Cournot oligopoly is mitigated by the fact that the marginal value of RE storage increases with the carbon tax. Carbon taxation unambiguously decreases thermal generation in both periods under Cournot oligopoly. Yet, somewhat counterintuitively, a carbon tax may increase peak thermal generation under perfect competition. Thus, the carbon tax depresses peak RE production less under Cournot oligopoly than under perfect competition, which means that the main finding of Bushnell (2003) may not hold with a carbon tax and in the presence of a generic storage device that is not $100 \%$ efficient. Investigating $\mathrm{RE}$ resource availability, we find that it decreases the marginal value of RE storage, which leads to more (less) RE (thermal) output with a greater impact in the peak period under both perfect competition and Cournot oligopoly. Finally, less efficient RE storage has contrasting effects on the marginal value of RE storage under perfect competition and Cournot oligopoly. A ceteris paribus degradation in efficiency monotonically increases the marginal value of RE storage under Cournot oligopoly as there is less (more) off-peak RE (thermal) production in order to preserve the RE resource for the peak. Under perfect competition, this result holds only if RE storage is relatively efficient, i.e., if RE storage is relatively inefficient, then a ceteris paribus degradation in its efficiency actually increases (decreases) off-peak RE (thermal) production and lowers storage value as it is not worthwhile to preserve the RE resource. Consequently, the RE producer's profit is non-monotonic in storage efficiency, i.e., the RE producer may actually be hurt by an infinitesimal increase in storage efficiency, which is in conflict with the social desirability of more efficient storage. We demonstrate that carbon policy may be used in this respect to align private and social incentives better.

The structure of this paper is as follows. Section 2 lays out the assumptions of our modelling framework and analytically solves for the industry equilibrium under the conjectures of perfect competition and Cournot oligopoly. Next, Section 3 conducts comparative statics of the solutions with respect to carbon policy, RE availability, and storage efficiency. Section 4 provides numerical examples to illustrate key results and to compare outcomes under perfect competition and Cournot oligopoly. Finally, Section 5 summarises the paper's contributions and indicates directions for future research. Appendices A-D contain the necessary and sufficient conditions for interior solutions (Downward et al., 2010), proofs of lemmata and propositions, a closed-loop Cournot analysis to assess the impact of dynamic decision making, and an investigation of a quadratic emission function, respectively. 


\section{Problem Formulation and Interior Solutions}

\subsection{Assumptions}

We assume a deterministic single-node, two-period power market with two types of profitmaximising producers, RE and thermal, with output $x_{j}$ [in MWh] and $y_{j}$ [in MWh], respectively, in each representative period, $j=1,2$. Each period, e.g., an hour, has inverse-demand function $P_{j}\left(x_{j}+y_{j}\right)=A_{j}-\left(x_{j}+y_{j}\right)\left[\right.$ in $\$ / \mathrm{MWh}$, where $A_{2}>A_{1}>0$, i.e., the price in period 2 is always greater than that in period 1 for a given total production level. The thermal producer has generation cost function $\frac{1}{2} C y_{j}^{2}$ [in $\$ / \mathrm{MWh}$ ], where $C>0$ [in $\$ / \mathrm{MWh}^{2}$ ] with emission rate $R>0$ [in $\mathrm{t} / \mathrm{MWh}]{ }^{1}$ Thermal production must pay a carbon tax of $T \geq 0$ [in $\left.\$ / \mathrm{t}\right]$. Note that "t" here is the SI abbreviation for "metric ton." By contrast, the RE producer has no cost of production and does not produce emissions from generation. However, its available stock of RE capacity is $D \geq 0$ [in $\mathrm{MWh}$, which must be fully used over the two periods. This assumption is similar to the one made for a hydropower producer by Bushnell (2003) because it cannot be seen to be "spilling" water by the regulator. Thus, the RE producer must determine $x_{j}$, i.e., how to allocate its stock, $D$, in each period. Unlike Bushnell (2003) and Crampes and Moreaux (2001), we allow for storage losses in order to reflect the characteristics of any resource-based RE output and not just hydropower (Sioshansi, 2014). In particular, the parameter $F \geq 1$ denotes the inefficiency of RE storage, i.e., generating $1 \mathrm{MWh}$ of RE output in period 2 requires a stock equivalent to $F \mathrm{MWh}$ of resource in period 1. Or, equivalently put, $\frac{1}{F}$ represents the efficiency of storage, i.e., every $1 \mathrm{MWh}$ of the RE resource not used in period 1 yields $\frac{1}{F}$ MWh of energy in period 2. Finally, in order to rule out corner solutions without RE or thermal generation in one or both periods, we further assume $A_{2}>F A_{1}$ and $C>3-2 \sqrt{2} \approx 0.17$.

The assumption $A_{2}>F A_{1}$ implies that period 2 is the peak period, which determines the sequencing of when $\mathrm{RE}$ is available and when peak consumption occurs. Indeed, in general, $\mathrm{RE}$ availability does not coincide with consumption, which necessitates the use of (inefficient) storage. This could represent a medium-term horizon if RE is hydropower with resource availability (water inflow) during the spring (off-peak) season and evaporation (losses) during the summer (peak) season (Førsund, 2015). Likewise, considering the short-term intraday patterns of solar availability, energy collected during the afternoon (off-peak) net load period needs to meet the peak net load in the evening, viz., the CAISO "duck curve" (CAISO, 2016). In terms of wind power, Germany experiences a similar intraday pattern in resource availability and consumption with more wind production during off-peak (early-morning) hours of a day (Rintamäki et al., 2017).

\footnotetext{
${ }^{1}$ Increasing marginal cost of production is not necessarily concomitant with an increasing marginal emission rate. For example, typical supply stacks for ERCOT in July 2015 (Global CCS Institute, 2011) and the U.K. during 20132014 (Timera Energy, 2013) are ordered nuclear, coal, natural gas, and oil, which exhibit increasing marginal costs but not necessarily increasing marginal emission rates. Nevertheless, we investigate the implication of an increasing marginal emission rate for the effects of a carbon tax in Appendix D.
} 


\subsection{Interior Solutions}

The RE producer's problem is to maximise its profit over the two periods consisting of revenue from sales subject to its resource availability:

$$
\begin{array}{cl}
\text { Maximise }_{x_{j} \geq 0} & {\left[A_{1}-\left(x_{1}+y_{1}\right)\right] x_{1}+\left[A_{2}-\left(x_{2}+y_{2}\right)\right] x_{2}} \\
\text { s.t. } & x_{1}+F x_{2}=D: \mu
\end{array}
$$

Here, $\mu$ [in $\$ / \mathrm{MWh}$ ] is the dual variable on the RE resource constraint representing the marginal value of RE storage. Since (1)-(2) is a convex optimisation problem, it may be replaced by its Karush-Kuhn-Tucker (KKT) conditions for optimality:

$$
\begin{array}{ll}
0 \leq x_{1} & \perp-\left[A_{1}-\left(2 x_{1}+y_{1}\right)\right]+\mu \geq 0 \\
0 \leq x_{2} & \perp-\left[A_{2}-\left(2 x_{2}+y_{2}\right)\right]+F \mu \geq 0 \\
\mu \text { free } & D-x_{1}-F x_{2}=0
\end{array}
$$

Note that if the RE producer is a price taker as in perfect competition $(\mathrm{PC})$, then it treats the price, $P_{j}\left(x_{j}+y_{j}\right)$, as given, thereby modifying KKT conditions (3)-(4) as follows:

$$
\begin{aligned}
& 0 \leq x_{1} \quad \perp-\left[A_{1}-\left(x_{1}+y_{1}\right)\right]+\mu \geq 0 \\
& 0 \leq x_{2} \quad \perp-\left[A_{2}-\left(x_{2}+y_{2}\right)\right]+F \mu \geq 0
\end{aligned}
$$

Likewise, the thermal producer's problem is to maximise its total profit, which consists of sales revenue, generation cost, and $\mathrm{CO}_{2}$ taxes:

$$
\text { Maximise }_{y_{j} \geq 0} \quad\left[A_{1}-\left(x_{1}+y_{1}\right)\right] y_{1}+\left[A_{2}-\left(x_{2}+y_{2}\right)\right] y_{2}-\frac{1}{2} C\left(y_{1}^{2}+y_{2}^{2}\right)-T R\left(y_{1}+y_{2}\right)
$$

Since (8) is a convex optimisation problem, it may be replaced by its KKT conditions for optimality:

$$
\begin{aligned}
& 0 \leq y_{1} \quad \perp-\left[A_{1}-\left(2 y_{1}+x_{1}\right)\right]+C y_{1}+T R \geq 0 \\
& 0 \leq y_{2} \quad \perp-\left[A_{2}-\left(2 y_{2}+x_{2}\right)\right]+C y_{2}+T R \geq 0
\end{aligned}
$$

Note from (9)-(10) that the carbon tax shifts the thermal producer's marginal cost curve up. Under PC, a price-taking thermal producer's KKT conditions become the following instead of (9)-(10):

$$
\begin{aligned}
& 0 \leq y_{1} \quad \perp-\left[A_{1}-\left(y_{1}+x_{1}\right)\right]+C y_{1}+T R \geq 0 \\
& 0 \leq y_{2} \quad \perp-\left[A_{2}-\left(y_{2}+x_{2}\right)\right]+C y_{2}+T R \geq 0
\end{aligned}
$$


In case of PC, we obtain the following interior solutions to (5)-(7) and (11)-(12): ${ }^{2}$

$$
\begin{aligned}
x_{1}^{P C} & =\frac{F\left(F A_{1}-A_{2}\right)+D+F(F-1) R T / C}{1+F^{2}} \\
x_{2}^{P C} & =\frac{A_{2}-F A_{1}+F D-(F-1) R T / C}{1+F^{2}} \\
y_{1}^{P C} & =\frac{A_{1}+F A_{2}-D-F(F-1) R T / C-\left(1+F^{2}\right) R T}{\left(1+F^{2}\right)(C+1)} \\
y_{2}^{P C} & =\frac{F\left(A_{1}+F A_{2}-D\right)+(F-1) R T / C-\left(1+F^{2}\right) R T}{\left(1+F^{2}\right)(C+1)} \\
\mu^{P C} & =\frac{C\left(A_{1}+F A_{2}-D\right)+(F+1) R T}{\left(1+F^{2}\right)(C+1)}
\end{aligned}
$$

Otherwise, under an open-loop Cournot oligopoly (CO), (3)-(5) and (9)-(10) yield the following interior solutions:

$$
\begin{aligned}
x_{1}^{C O}= & \frac{F\left(F A_{1}-A_{2}\right)(C+1)+F(F-1) R T}{\left(1+F^{2}\right)(2 C+3)}+\frac{D}{1+F^{2}} \\
x_{2}^{C O}= & \frac{\left(A_{2}-F A_{1}\right)(C+1)-(F-1) R T}{\left(1+F^{2}\right)(2 C+3)}+\frac{F D}{1+F^{2}} \\
y_{1}^{C O}= & \frac{(2 C+3) A_{1}+F^{2}(C+2) A_{1}+F(C+1) A_{2}-(2 C+3) D}{\left(1+F^{2}\right)(C+2)(2 C+3)} \\
& -\frac{\left[2 C+3-F+F^{2}(2 C+4)\right] R T}{\left(1+F^{2}\right)(C+2)(2 C+3)} \\
y_{2}^{C O}= & \frac{F(C+1) A_{1}+(C+2) A_{2}+F^{2}(2 C+3) A_{2}-F(2 C+3) D}{\left(1+F^{2}\right)(C+2)(2 C+3)} \\
& -\frac{\left[2 C+4-F+F^{2}(2 C+3)\right] R T}{\left(1+F^{2}\right)(C+2)(2 C+3)} \\
\mu^{C O}= & \frac{(C+1) A_{1}+F(C+1) A_{2}-(2 C+3) D+(F+1) R T}{\left(1+F^{2}\right)(C+2)}
\end{aligned}
$$

Here, both off-peak and peak production decisions are treated as if they were made together, which means that there are no first- and second-stage decision variables. By contrast, a closed-loop (CL) Cournot framework takes into account the response of the thermal producer in period 2 due to any transfer of RE resource from period 1 to period 2 (Crampes and Moreaux, 2001). In order to examine the consequences of dynamic decision making, e.g., necessitated by (possibly random) shocks to the RE stock, $D$, for our general findings, we formulate in Appendix C a CL Cournot framework, i.e., a subgame perfect Nash equilibrium, with first- and second-stage decisions.

\footnotetext{
${ }^{2}$ Sufficient conditions for the equilibria to be interior are provided in Appendix A.
} 


\section{Comparative Statics}

\subsection{Impact of Carbon Policy}

We first analyse the impact of carbon policy, $T$, on interior solutions under PC as summarised by the following proposition: ${ }^{3}$

Proposition 1. Differentiating (13)-(17) with respect to $T$, we find that the following results are unconditional for any interior solution:

$$
\frac{\partial x_{1}^{P C}}{\partial T}>0, \frac{\partial x_{2}^{P C}}{\partial T}<0,\left|\frac{\partial x_{1}^{P C}}{\partial T}\right|>\left|\frac{\partial x_{2}^{P C}}{\partial T}\right|, \frac{\partial y_{1}^{P C}}{\partial T}<0, \frac{\partial \mu^{P C}}{\partial T}>0 .
$$

Meanwhile, the following finding for any interior solution is conditional on the value of $C$ :

$$
\frac{\partial y_{2}^{P C}}{\partial T}>0 \text { if } C<\frac{F-1}{1+F^{2}}
$$

Overall, the results are generally intuitive since the carbon tax increases the marginal cost of thermal production, which increases the marginal value of RE and shifts RE production to period 1 and curbs overall thermal output. However, increasing the carbon tax may have the counterproductive effect of increasing peak carbon-intensive production. This effect holds if the thermal marginal cost is relatively low with respect to the storage efficiency of the carbon-free resource, i.e., the upper bound on $C$ is necessary only for the somewhat counterintuitive finding in Proposition 1. In other words, if the tax applies to a baseload type of plant, e.g., combined-cycle gas turbines or coal-fired power plants, and storage is relatively inefficient, then a carbon policy provides the counterproductive incentive to increase thermal generation during the peak period. ${ }^{4}$

In order to explain why a carbon tax may increase period-2 thermal generation, we perform a decomposition of the comparative statics at equilibrium (Bulow et al., 1985). Specifically, we start with the optimality conditions for perfect competition, where each (implicit) variable is a function of the other (explicit) variables as obtained from KKT conditions (5)-(7) and (11)-(12):

$$
\begin{aligned}
X_{1}^{P C}\left(y_{1}, y_{2}\right) & =A_{1}-y_{1}-M^{P C}\left(y_{1}, y_{2}\right) \\
X_{2}^{P C}\left(y_{1}, y_{2}\right) & =A_{2}-y_{2}-F M^{P C}\left(y_{1}, y_{2}\right) \\
Y_{1}^{P C}\left(x_{1}\right) & =\frac{A_{1}-T R-x_{1}}{C+1} \\
Y_{2}^{P C}\left(x_{2}\right) & =\frac{A_{2}-T R-x_{2}}{C+1} \\
M^{P C}\left(y_{1}, y_{2}\right) & =\frac{A_{1}-y_{1}+F A_{2}-F y_{2}-D}{1+F^{2}}
\end{aligned}
$$

\footnotetext{
${ }^{3}$ All proofs are in Appendix B.

${ }^{4}$ Given the assumption $C>3-2 \sqrt{2}$, for $\frac{\partial y_{2}^{P C}}{\partial T}$ to be positive, it is necessary that $\frac{F-1}{1+F^{2}}>3-2 \sqrt{2}$. This inequality implies that $F \in\left(\frac{1-\sqrt{56 \sqrt{2}-79}}{2(3-2 \sqrt{2})}, \frac{1+\sqrt{56 \sqrt{2}-79}}{2(3-2 \sqrt{2})}\right) \approx(1.6,4.2)$.
} 
In (23)-(27), implicit variables such as $X_{1}^{P C}$ and $X_{2}^{P C}$ depend directly on $y_{1}$ and $y_{2}$ as well as on $\mu$, which also depends on $y_{1}$ and $y_{2}$. Thus, in order to trace the total impact of a change in $T$ on $x_{1}^{P C}$, we must account for (i) the direct effect of $T$ on $x_{1}^{P C}$, (ii) the indirect effect of $T$ on $x_{1}^{P C}$ via $y_{1}$, and (iii) the indirect effect of $T$ on $x_{1}^{P C}$ via $\mu$. Indeed, this is the purpose of the following decomposition conducted by differentiating these functions with respect to $T$ and evaluating the resulting expressions at the equilibrium of the explicit variables, $\Omega^{P C}$ :

$$
\left[\begin{array}{ccccc}
1 & 0 & 1 & 0 & 1 \\
0 & 1 & 0 & 1 & F \\
\frac{1}{C+1} & 0 & 1 & 0 & 0 \\
0 & \frac{1}{C+1} & 0 & 1 & 0 \\
0 & 0 & \frac{1}{1+F^{2}} & \frac{F}{1+F^{2}} & 1
\end{array}\right]\left[\begin{array}{c}
\left.\frac{\partial X_{1}^{P C}}{\partial T}\right|_{\Omega^{P C}} \\
\left.\frac{\partial X_{2}^{P C}}{\partial T}\right|_{\Omega^{P C}} \\
\left.\frac{\partial Y_{1}^{P C}}{\partial T}\right|_{\Omega^{P C}} \\
\left.\frac{\partial Y_{2}^{P C}}{\partial T}\right|_{\Omega^{P C}} \\
\left.\frac{\partial M^{P C}}{\partial T}\right|_{\Omega^{P C}}
\end{array}\right]=\left[\begin{array}{c}
0 \\
0 \\
-\frac{R}{C+1} \\
-\frac{R}{C+1} \\
0
\end{array}\right]
$$

In (28), the vector on the right-hand side comprises the direct effects of the carbon tax, which impact only thermal production. The matrix on the left-hand side contains all of the indirect effects of the carbon tax.

Intuitively, an increase in the carbon tax directly affects thermal generation by increasing its marginal cost of production as observed in the third and fourth entries of the right-hand side vector in (28). This change in market conditions has the indirect effect of increasing the residual demand faced by the RE producer in both periods, thereby inducing an increase in its generation in the short term. However, there is also an intertemporal indirect effect, which stems from the higher marginal value of the renewable resource and leads to lower RE output. Overall, the short-term indirect effect dominates, thereby leading to an increase in period-1 RE output and a corresponding decrease in period-2 RE output. Consequently, the possibility of an increase in period-2 thermal production due to the carbon tax depends on both the relative inefficiency of RE storage ( $F$ high) and the efficiency of thermal production ( $C$ low). Indeed, the indirect effect of the carbon tax in shifting RE production to period 1 is intensified if $F$ is high, which increases the price in period 2 drastically. At the same time, increasing thermal generation at low cost in period 2 as a response to an increase in the marginal value of RE may lead to the somewhat perverse outcome that thermal generation may actually increase overall in period 2 .

We likewise investigate the role of the carbon tax on $\mathrm{CO}$ production decisions and the RE storage value:

Proposition 2. Differentiating (18)-(22) with respect to $T$, we obtain the following unconditional results for any interior solution:

$$
\frac{\partial x_{1}^{C O}}{\partial T}>0, \frac{\partial x_{2}^{C O}}{\partial T}<0,\left|\frac{\partial x_{1}^{C O}}{\partial T}\right|>\left|\frac{\partial x_{2}^{C O}}{\partial T}\right|, \frac{\partial y_{1}^{C O}}{\partial T}<\frac{\partial y_{2}^{C O}}{\partial T}<0, \frac{\partial \mu^{C O}}{\partial T}>0 .
$$

These findings are intuitive and mirror those from PC except that period-2 thermal output unambiguously decreases with the carbon tax. Overall, period-1 RE production increases by more than 
period-2 RE production decreases, and the impact of the carbon tax is more severe on period-1 thermal output than that in period 2. Finally, by increasing the RE storage value, the carbon tax alleviates the propensity of $\mu^{C O}$ to become negative, thereby potentially reversing the Bushnell (2003) finding.

We decompose the comparative statics for $\mathrm{CO}$ via the same steps as those for PC where each (implicit) variable is a function of the other (explicit) variables as obtained from the KKT conditions (3)-(5) and (9)-(10):

$$
\begin{aligned}
X_{1}^{C O}\left(y_{1}, y_{2}\right) & =\frac{A_{1}-y_{1}-M^{C O}\left(y_{1}, y_{2}\right)}{2} \\
X_{2}^{C O}\left(y_{1}, y_{2}\right) & =\frac{A_{2}-y_{2}-F M^{C O}\left(y_{1}, y_{2}\right)}{2} \\
Y_{1}^{C O}\left(x_{1}\right) & =\frac{A_{1}-T R-x_{1}}{C+2} \\
Y_{2}^{C O}\left(x_{2}\right) & =\frac{A_{2}-T R-x_{2}}{C+2} \\
M^{C O}\left(y_{1}, y_{2}\right) & =\frac{A_{1}-y_{1}+F A_{2}-F y_{2}-2 D}{1+F^{2}}
\end{aligned}
$$

We next differentiate these functions with respect to $T$ and evaluate the resulting expressions at the equilibrium of the explicit variables, $\Omega^{C O}$ :

$$
\left[\begin{array}{ccccc}
1 & 0 & \frac{1}{2} & 0 & \frac{1}{2} \\
0 & 1 & 0 & \frac{1}{2} & \frac{F}{2} \\
\frac{1}{C+2} & 0 & 1 & 0 & 0 \\
0 & \frac{1}{C+2} & 0 & 1 & 0 \\
0 & 0 & \frac{1}{1+F^{2}} & \frac{F}{1+F^{2}} & 1
\end{array}\right]\left[\begin{array}{c}
\left.\frac{\partial X_{1}^{C O}}{\partial T}\right|_{\Omega^{C O}} \\
\left.\frac{\partial X_{2}^{C O}}{\partial T}\right|_{\Omega^{C O}} \\
\left.\frac{\partial Y_{1}^{C O}}{\partial T}\right|_{\Omega^{C O}} \\
\left.\frac{\partial Y_{2}^{C O}}{\partial T}\right|_{\Omega^{C O}} \\
\left.\frac{\partial M^{C O}}{\partial T}\right|_{\Omega^{C O}}
\end{array}\right]=\left[\begin{array}{c}
0 \\
0 \\
-\frac{R}{C+2} \\
-\frac{R}{C+2} \\
0
\end{array}\right]
$$

Above a certain carbon-tax threshold, the RE producer's incentive to generate more in period 1 under $\mathrm{CO}$ vis-à-vis $\mathrm{PC}$ is mitigated by the fact that the marginal value of $\mathrm{RE}$ storage increases with the carbon tax (Proposition 2). This unambiguously decreases thermal generation in both periods under CO but makes it possible for period-2 thermal generation to increase under PC. Indeed, under $\mathrm{CO}$, the thermal producer optimally sets its marginal cost equal to the marginal revenue instead of to the price and exercises a price markup by withholding generation even during the peak period. Consequently, the carbon tax depresses period-2 RE production more under PC than under CO, which means that the main finding of Bushnell (2003), i.e., that market power induces more off-peak $\mathrm{RE}$ resource allocation than under PC, may not hold under a relatively high carbon tax.

Proposition 3. Comparing (18) and (13), off-peak RE generation under $C O$ exceeds that under $P C$ only if $T<\frac{\left(A_{2}-F A_{1}\right)(C+2) C}{R(F-1)(C+3)}$ for any interior solution. 


\subsection{Impact of Storage Capacity}

We now investigate how PC solutions are affected by RE storage capacity (or, equivalently, RE availability), $D$, which is likely to be a factor as policies to support RE will similarly spur development of energy storage:

Proposition 4. Differentiating (13)-(17) with respect to D, we obtain the following unconditional results for any interior solution:

$$
\frac{\partial x_{2}^{P C}}{\partial D}>\frac{\partial x_{1}^{P C}}{\partial D}>0, \frac{\partial y_{2}^{P C}}{\partial D}<\frac{\partial y_{1}^{P C}}{\partial D}<0, \frac{\partial \mu^{P C}}{\partial D}<0
$$

The results are generally intuitive: more $\mathrm{RE}$ resource availability increases $\mathrm{RE}$ output in both periods, decreases thermal output in both periods, and lowers the RE storage value. Moreover, RE output increases more in period 2 than it does in period 1, while thermal output decreases more in period 2 than it does in period 1. Finally, the increase in RE output in each period more than offsets the corresponding reduction in thermal generation.

In order to decompose these findings, we next differentiate $(23)-(27)$ with respect to $D$ and evaluate the resulting expressions at the equilibrium of the explicit variables:

$$
\left[\begin{array}{ccccc}
1 & 0 & 1 & 0 & 1 \\
0 & 1 & 0 & 1 & F \\
\frac{1}{C+1} & 0 & 1 & 0 & 0 \\
0 & \frac{1}{C+1} & 0 & 1 & 0 \\
0 & 0 & \frac{1}{1+F^{2}} & \frac{F}{1+F^{2}} & 1
\end{array}\right]\left[\begin{array}{c}
\left.\frac{\partial X_{1}^{P C}}{\partial D}\right|_{\Omega^{P C}} \\
\left.\frac{\partial X_{2}^{P C}}{\partial D}\right|_{\Omega^{P C}} \\
\left.\frac{\partial Y_{1}^{P C}}{\partial D}\right|_{\Omega^{P C}} \\
\left.\frac{\partial Y_{2}^{P C}}{\partial P C}\right|_{\Omega^{P C}}
\end{array}\right]=\left[\begin{array}{c}
0 \\
0 \\
0 \\
0 \\
-\frac{1}{1+F^{2}}
\end{array}\right]
$$

The direct effect of increasing the stored RE is to reduce RE's shadow price, which is the final entry of the right-hand side vector in (35). This has the short-term indirect effect of reducing thermal generation (increasing RE output) as given by the first and second entries in the third and fourth rows (third and fourth entries in the first and second rows), respectively, of the left-hand side matrix in (35). In turn, the intertemporal impact of higher $D$, i.e., a lower shadow price, further expands $\mathrm{RE}$ output, which is reflected in the final entries of the first and second rows of the left-hand side matrix in (35).

Turning to the impact of RE resource availability under CO, we have:

Proposition 5. Differentiating (18)-(22) with respect to D, we obtain the following unconditional results for any interior solution:

$$
\frac{\partial x_{2}^{C O}}{\partial D}>\frac{\partial x_{1}^{C O}}{\partial D}>0, \frac{\partial y_{2}^{C O}}{\partial D}<\frac{\partial y_{1}^{C O}}{\partial D}<0, \frac{\partial \mu^{C O}}{\partial D}<0
$$

Again, the results here follow on from those under PC. In fact, the impact of $D$ on RE production, $\frac{\partial x_{1}^{C O}}{\partial D}$ and $\frac{\partial x_{2}^{C O}}{\partial D}$, is the same under $\mathrm{CO}$ as under $\mathrm{PC}$ with a greater impact on period-2 production. 
The magnitudes for the impact of $D$ on thermal production, $\frac{\partial y_{1}^{C O}}{\partial D}$ and $\frac{\partial y_{2}^{C O}}{\partial D}$, are lower than under $\mathrm{PC}$ because thermal generation was already restricted due to the exertion of market power. As under PC, there is a greater impact on period-2 thermal production. Likewise, the reduction in each period's thermal generation is more than offset by the corresponding increase in RE output. Finally, although more $\mathrm{RE}$ resource availability reduces the shadow price of $\mathrm{RE}$ storage, $\frac{\partial \mu^{C O}}{\partial D}$, its impact is stronger under $\mathrm{CO}$ than under $\mathrm{PC}$ due to the RE producer's desire to reduce output.

We next differentiate (29)-(33) with respect to $D$ and evaluate the resulting expressions at the equilibrium of the explicit variables in order to decompose the comparative statics:

$$
\left[\begin{array}{ccccc}
1 & 0 & \frac{1}{2} & 0 & \frac{1}{2} \\
0 & 1 & 0 & \frac{1}{2} & \frac{F}{2} \\
\frac{1}{C+2} & 0 & 1 & 0 & 0 \\
0 & \frac{1}{C+2} & 0 & 1 & 0 \\
0 & 0 & \frac{1}{1+F^{2}} & \frac{F}{1+F^{2}} & 1
\end{array}\right]\left[\begin{array}{c}
\left.\frac{\partial X_{1}^{C O}}{\partial D}\right|_{\Omega^{C O}} \\
\left.\frac{\partial X_{2}^{C O}}{\partial D}\right|_{\Omega^{C O}} \\
\left.\frac{\partial Y_{1}^{C O}}{\partial D}\right|_{\Omega^{C O}} \\
\left.\frac{\partial Y_{2}^{C O}}{\partial D}\right|_{\Omega^{C O}} \\
\left.\frac{\partial M^{C O}}{\partial D}\right|_{\Omega^{C O}}
\end{array}\right]=\left[\begin{array}{c}
0 \\
0 \\
0 \\
0 \\
-\frac{2}{1+F^{2}}
\end{array}\right]
$$

It may be observed that the direct effect of $D$ on the marginal value of $\mathrm{RE}$ is stronger under $\mathrm{CO}$ than under PC (final entry of the right-hand side vector in (36)). Conversely, the intertemporal effects of $D$ on RE output are lower under CO than under PC (final entries in the first and second rows of the left-hand side matrix in (36)).

\subsection{Impact of Storage Efficiency}

As more energy storage is deployed, the efficiency of new devices is likely to improve. At the same time, the need for storage may encourage owners of superannuated devices to continue their operation, which could cause a degradation of storage efficiency. We, therefore, conduct comparative statics first on the effects of $F$ on the PC solution under the assumption of $T=0$ and any $D \in \mathcal{S}^{P C} \cap \mathcal{S}_{\mu}^{C O}:^{5}$

Proposition 6. Differentiating (13)-(17) with respect to $F$ for $T=0$, we obtain the following unconditional results for any interior solution:

$$
\frac{\partial x_{2}^{P C}}{\partial F}<0, \frac{\partial y_{2}^{P C}}{\partial F}>0
$$

Meanwhile, the following findings for $T=0$ are all conditional on the value of $F$ for any interior solution:

$$
\frac{\partial x_{1}^{P C}}{\partial F}<0, \frac{\partial y_{1}^{P C}}{\partial F}>0, \frac{\partial \mu^{P C}}{\partial F}>0 \text { if } F<\frac{\left(D-A_{1}\right)+\sqrt{A_{2}^{2}+\left(D-A_{1}\right)^{2}}}{A_{2}} .
$$

\footnotetext{
${ }^{5}$ This effectively means $D \in\left[\max \left\{F\left(A_{2}-F A_{1}\right), \frac{\left(A_{1}+F A_{2}\right)(C+1)}{2 C+3}\right\}, \frac{A_{1}(C+2)\left(F^{2}+1\right)+\left(A_{1}+F A_{2}\right)(C+1)}{2 C+3}\right]$ from (A-5a) and $(\mathrm{A}-5 \mathrm{~b})$.
} 
The signs for $\frac{\partial x_{1}^{P C}}{\partial F}, \frac{\partial y_{1}^{P C}}{\partial F}$, and $\frac{\partial \mu^{P C}}{\partial F}$ are negative, positive, and positive, respectively, as long as $F<\frac{\left(D-A_{1}\right)+\sqrt{A_{2}^{2}+\left(D-A_{1}\right)^{2}}}{A_{2}}$. Thus, if RE storage is not "too inefficient," then a ceteris paribus degradation of storage efficiency will lead to less (more) period-1 RE (thermal) production and a higher storage value in order to preserve the RE resource for period 2. By contrast, for relatively high $F$, i.e., "inefficient storage," degradation of RE storage efficiency will actually increase (decrease) period-1 RE (thermal) production and lower the storage value as it is not worthwhile to preserve the $\mathrm{RE}$ resource for period 2. The critical threshold for this effect is $F>\frac{\left(D-A_{1}\right)+\sqrt{A_{2}^{2}+\left(D-A_{1}\right)^{2}}}{A_{2}}$. Finally, from (B-27) and (B-29), degradation of RE storage efficiency decreases period-2 RE production and increases period-2 thermal production, respectively.

We next differentiate (23)-(27) with respect to $F$ for $T=0$ and evaluate the resulting expressions at the equilibrium of the explicit variables in order to explain these comparative statics further:

$$
\left[\begin{array}{ccccc}
1 & 0 & 1 & 0 & 1 \\
0 & 1 & 0 & 1 & F \\
\frac{1}{C+1} & 0 & 1 & 0 & 0 \\
0 & \frac{1}{C+1} & 0 & 1 & 0 \\
0 & 0 & \frac{1}{1+F^{2}} & \frac{F}{1+F^{2}} & 1
\end{array}\right]\left[\begin{array}{c}
\left.\frac{\partial X_{1}^{P C}}{\partial F}\right|_{\Omega^{P C}} \\
\left.\frac{\partial X_{2}^{P C}}{\partial F}\right|_{\Omega^{P C}} \\
\left.\frac{\partial Y_{1}^{P C}}{\partial F}\right|_{\Omega^{P C}} \\
\left.\frac{\partial Y_{2}^{P C}}{\partial M^{P C}}\right|_{\Omega^{P C}}
\end{array}\right]=\left[\begin{array}{c}
0 \\
-M^{P C} \\
0 \\
0 \\
\frac{\left(A_{2}-F A_{1}\right)-F\left(F A_{2}+A_{1}\right)+2 F D+2 F Y_{1}^{P C}+\left(F^{2}-1\right) Y_{2}^{P C}}{\left(1+F^{2}\right)^{2}}
\end{array}\right]
$$

If RE storage is not "too inefficient," then the direct effect of decreasing the efficiency of RE storage is to increase RE's shadow price, which is the last term of the right-hand side vector of (37). This has the short-term indirect effect of reducing RE generation (increasing thermal output) as given by the third and fourth entries in the first and second rows (the first and second entries in the third and fourth rows), respectively, of the left-hand side matrix in (37). In turn, the intertemporal impact of higher $F$ further decreases RE output, which is reflected in the final terms of the first and second rows of the left-hand side matrix in (37) as well as the RE shadow price as given in the third and fourth terms of the final row of the left-hand side matrix in (37). Intuitively, the opportunity cost of RE storage increases overall with storage inefficiency when period-2 demand is relatively large because the RE producer would like to sell more period- 2 energy but is unable to do so due to efficiency losses. Hence, if RE storage is relatively inefficient, then a ceteris paribus degradation in its efficiency actually increases (decreases) period-1 RE (thermal) production and lowers $\mathrm{RE}$ storage value as it is not worthwhile to preserve the RE resource.

Moving on to the $\mathrm{CO}$ case, we obtain the following result concerning the impact of $F$ :

Proposition 7. Differentiating (18)-(22) with respect to $F$ for $T=0$, we obtain the following unconditional results for any interior solution:

$$
\frac{\partial x_{1}^{C O}}{\partial F}<0, \frac{\partial x_{2}^{C O}}{\partial F}<0, \frac{\partial y_{1}^{C O}}{\partial F}>0, \frac{\partial y_{2}^{C O}}{\partial F}>0, \frac{\partial \mu^{C O}}{\partial F}>0
$$

Thus, a ceteris paribus degradation of storage efficiency leads to less (more) period-1 RE (thermal) 
production and a higher storage value in order to preserve the RE resource for period 2. This mirrors the PC outcome for relatively efficient RE storage. Indeed, with production withholding due to market power, the marginal value of RE storage strictly decreases with greater storage efficiency.

We next differentiate (29)-(33) with respect to $F$ for $T=0$ and evaluate the resulting expressions at the equilibrium of the explicit variables in order to decompose the comparative statics:

$$
\left[\begin{array}{ccccc}
1 & 0 & \frac{1}{2} & 0 & \frac{1}{2} \\
0 & 1 & 0 & \frac{1}{2} & \frac{F}{2} \\
\frac{1}{C+2} & 0 & 1 & 0 & 0 \\
0 & \frac{1}{C+2} & 0 & 1 & 0 \\
0 & 0 & \frac{1}{1+F^{2}} & \frac{F}{1+F^{2}} & 1
\end{array}\right]\left[\left.\begin{array}{c}
\left.\frac{\partial X_{1}^{C O}}{\partial F}\right|_{\Omega^{C O}} \\
\left.\frac{\partial X_{2}^{C O}}{\partial F}\right|_{\Omega^{C O}} \\
\left.\frac{\partial Y_{1}^{C O}}{\partial F}\right|_{\Omega^{C O}} \\
\left.\frac{\partial Y_{2}^{C O}}{\partial F}\right|_{\Omega^{C O}} \\
\frac{\partial M^{C O}}{\partial F}
\end{array}\right|_{\Omega^{C O}}\right]=\left[\begin{array}{c}
0 \\
-\frac{1}{2} M^{C O} \\
0 \\
0 \\
\frac{\left(A_{2}-F A_{1}\right)-F\left(F A_{2}+A_{1}\right)+4 F D+2 F Y_{1}^{C O}+\left(F^{2}-1\right) Y_{2}^{C O}}{\left(1+F^{2}\right)^{2}}
\end{array}\right]
$$

The direct effect of efficiency degradation is stronger under CO as opposed to PC. However, the intertemporal effects are lower in magnitude. Finally, a ceteris paribus degradation in efficiency monotonically increases the marginal value of RE storage under Cournot oligopoly as there is less (more) period-1 RE (thermal) production and a higher RE storage value in order to preserve the $\mathrm{RE}$ resource for period 2 .

\section{Numerical Examples}

We illustrate the analytical results from Sections 2 and 3 by performing numerical examples using the base-case data in Table 1. These parameter values are selected to be in the set of interior solutions, $\mathcal{S}$, as defined in Lemma 3 and are varied with respect to $T, D$, and $F$ in order to gain insights about these parameters' effects on industry equilibrium, the potential for the exercise of market power, and the marginal value of RE.

Table 1: Data for numerical examples

\begin{tabular}{ll}
\hline Parameter & Value \\
\hline$A_{1}$ & 125 \\
$A_{2}$ & 250 \\
$D$ & 250 \\
$C$ & 0.18 \\
$F$ & 1.80 \\
$R$ & 1.0 \\
$T$ & 20 \\
\hline
\end{tabular}

\subsection{Impact of Carbon Tax}

We first investigate the impact of the carbon tax by letting it vary between $\$ 0 / \mathrm{t}$ and $\$ 25 / \mathrm{t}$. First, we note in Figure 1 that a ceteris paribus increase in $T$ increases the marginal value of $\mathrm{RE}$ 
under both $\mathrm{PC}$ and $\mathrm{CO}$ as indicated in Propositions 1 and 2. Moreover, if the carbon tax were higher than $\$ 57.68 / \mathrm{t}$ as indicated by (A-4a) in Lemma 2 , then $\mu^{C O}$ would no longer be negative, i.e., reversing the result of Bushnell (2003). Next, Figure 2 sketches out the results of Propositions 1 and 2 concerning RE generation. Note that the main finding of Bushnell (2003) regarding $x_{1}^{C O}>x_{1}^{P C}$ is reversed when $T$ exceeds $\$ 3.86 / \mathrm{t}$ as indicated in Proposition 3.

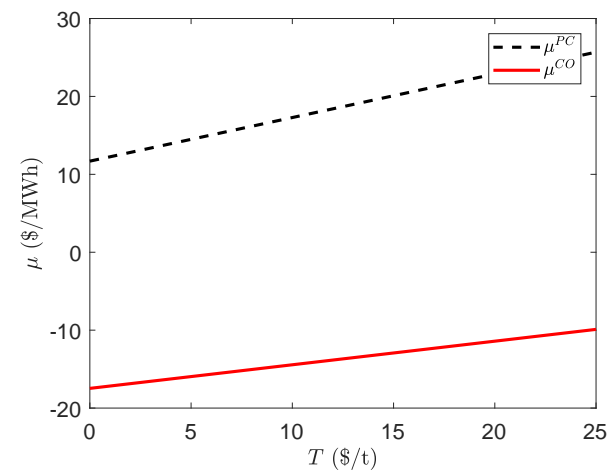

Figure 1: Impact of carbon tax on RE marginal value

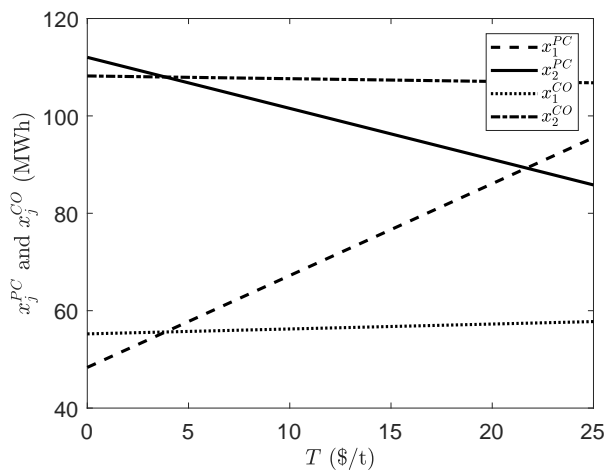

Figure 2: Impact of carbon tax on RE production

Figure 3 illustrates the behaviour of thermal generation as shown in Propositions 1 and 2. The somewhat surprising result that $y_{2}^{P C}$ may actually increase with $T$ is shown here and holds as long as $C<0.1887$. Finally, Figures 4-6 illustrate the generally intuitive findings concerning the impact of carbon taxation on producers' profit, carbon emissions, and social welfare. ${ }^{6}$

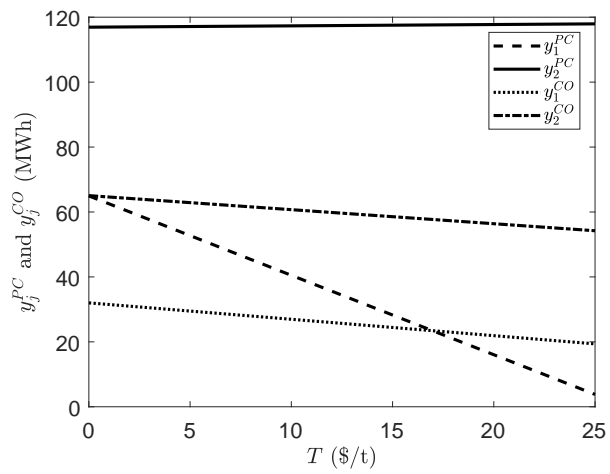

Figure 3: Impact of carbon tax on thermal production

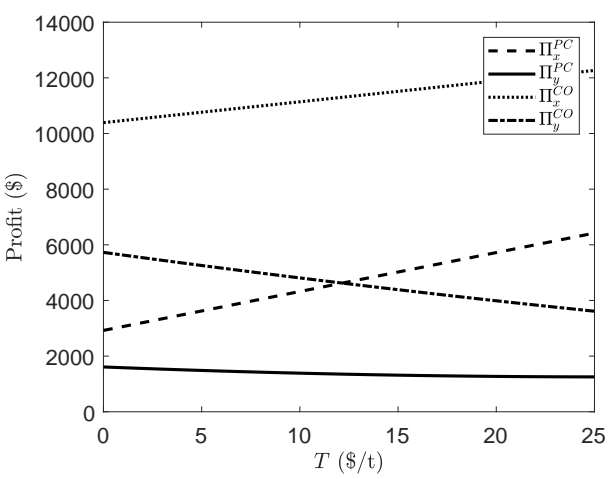

Figure 4: Impact of carbon tax on profit

\subsection{Impact of RE Availability}

Next, we explore the impact of RE availability by letting it vary between $220 \mathrm{MWh}$ and 300 MWh. Figure 7 demonstrates that a ceteris paribus increase in $D$ decreases the marginal value of RE under both PC and $\mathrm{CO}$ as indicated in Propositions 4 and 5. A value of RE availability lower than

\footnotetext{
${ }^{6}$ We numerically investigate the robustness of the findings in Propositions 1 and 3 with respect to a quadratic emission function in Appendix D.
} 


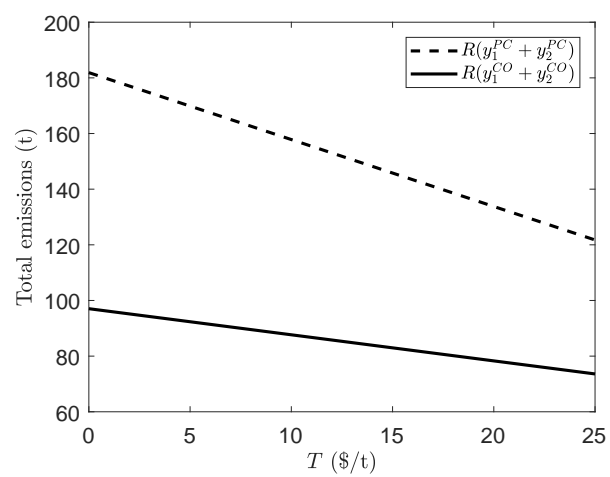

Figure 5: Impact of carbon tax on carbon emissions

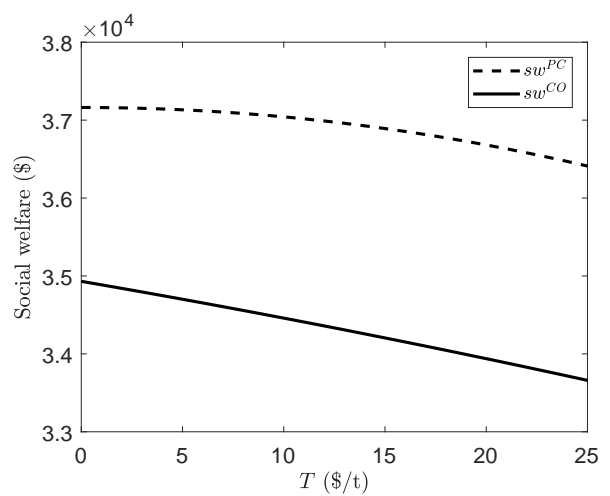

Figure 6: Impact of carbon tax on social welfare

218.60 MWh (as indicated by (A-4a) in Lemma 2) would render $\mu^{C O}$ non-negative contrary to the finding of Bushnell (2003). Figure 8 sketches out the results of Propositions 4 and 5 concerning RE generation, i.e., more RE availability leads to more RE generation. Likewise, more RE availability lowers thermal production, increases (decreases) the profit of the RE producer (thermal producer), decreases emissions, and increases social welfare as indicated in Figures 9-12.

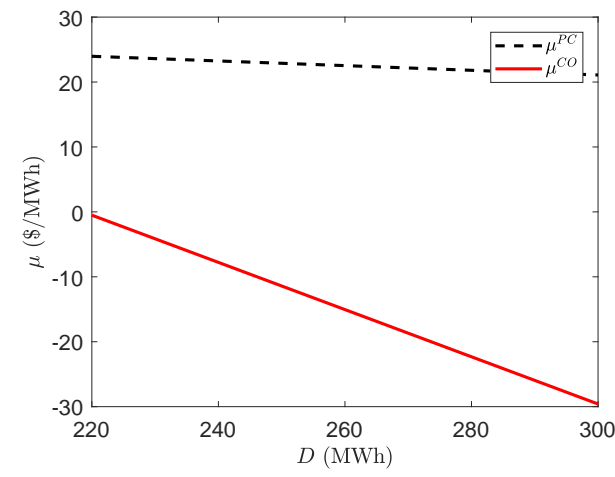

Figure 7: Impact of $\mathrm{RE}$ availability on $\mathrm{RE}$ marginal value

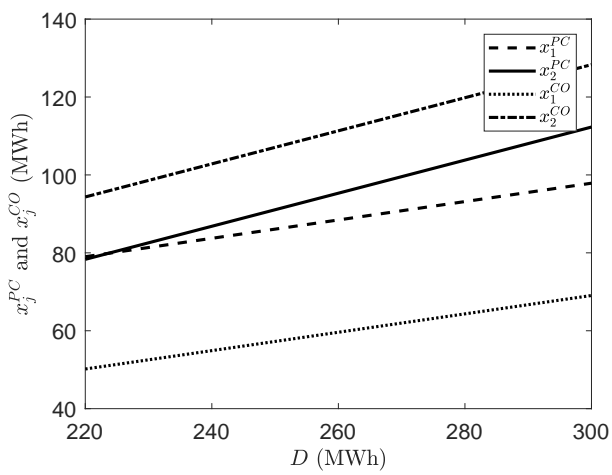

Figure 8: Impact of RE availability on RE production

\subsection{Impact of RE Storage Efficiency}

Given the expected development of energy storage technologies in order to back up RE deployment mandated by policy, an investigation of storage efficiency on market equilibria is desirable to support regulatory authorities. Here, we illustrate numerically the analytical comparative statics derived for $T=0$ in Section 3.3 when $F$ varies from 1 to 2, i.e., between $50 \%$ and $100 \%$ efficiency. Furthermore, we also sketch out the behaviour of key variables for $T=20$ in order to gain insights that are not possible to obtain analytically.

First, Figure 13 demonstrates that for $T=0, \mu^{P C}$ behaves non-monotonically as proven in Proposition 6: a degradation in storage efficiency initially increases the marginal value of RE storage but then causes it to diminish. The critical point is at $F=\frac{\left(D-A_{1}\right)+\sqrt{A_{2}^{2}+\left(D-A_{1}\right)^{2}}}{A_{2}}$, which is 1.618 for our parameter values. Intuitively, storage capacity is worth expanding in response to an infinitesimal 


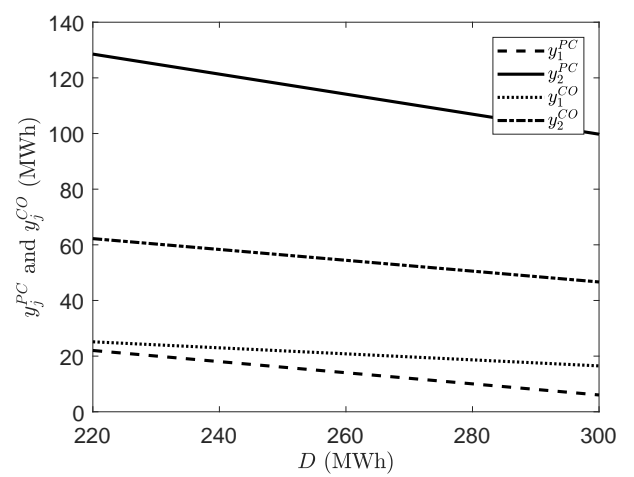

Figure 9: Impact of RE availability on thermal production

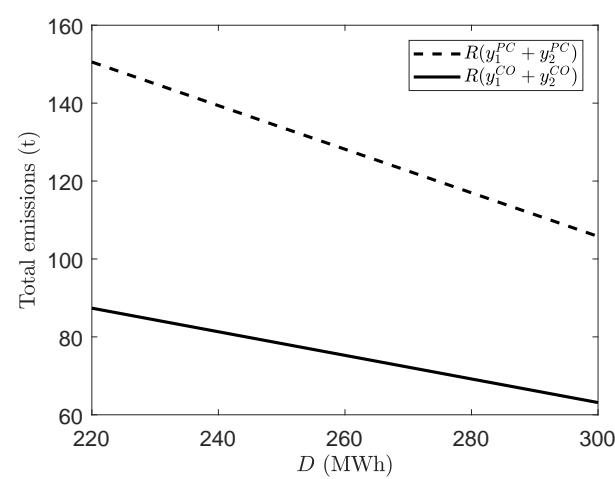

Figure 11: Impact of RE availability on carbon emissions

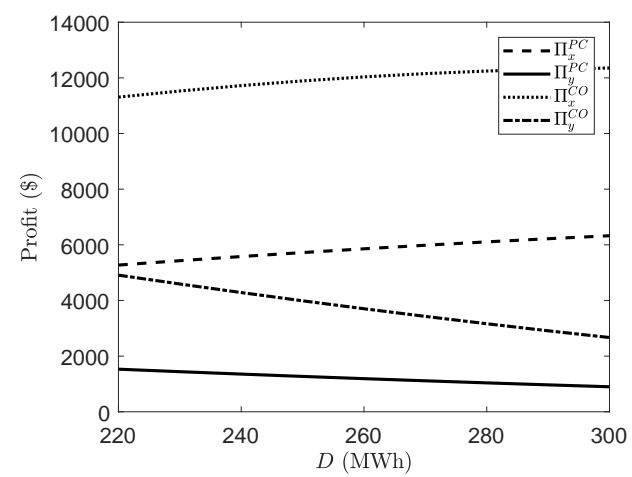

Figure 10: Impact of RE availability on profit

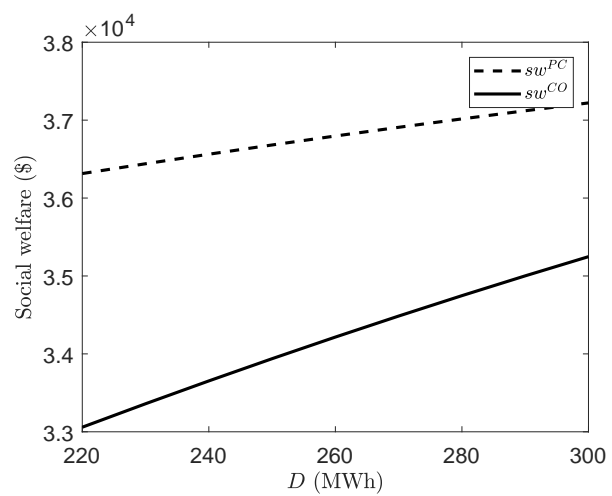

Figure 12: Impact of RE availability on social welfare

loss in efficiency only if the device is still relatively efficient. The same figure reveals a similar pattern in $\mu^{P C}$ 's behaviour with respect to $F$ even for $T>0$. However, the "turning point" occurs for a much lower value of $F=1.05$. In effect, the RE producer is willing to tolerate a much narrower range in RE storage efficiency for which its marginal valuation is positive with respect to $F$ as the carbon tax already forces it to shift its stock to period 1, i.e., the need to expand storage is obviated by carbon policy. By contrast, Figure 14 indicates the monotonic behaviour of $\mu^{C O}$ with respect to $F$ for both $T=0$ (as indicated by Proposition 7 ) and $T>0$. The only difference between the two curves is that the imposition of a carbon tax increases the marginal value of RE storage.

Next, Figures 15 and 16 illustrate how RE production is affected by storage degradation for $T=0$ and $T=20$, respectively. Again, the analytical results of Propositions 6 and 7 are revealed to hold for $T>0$ including the non-monotonic behaviour of $x_{1}^{P C}$ : indeed, for $F<1.618$, it decreases with $F$ for $T=0$ before increasing (Figure 15). The explanation for this non-monotonicity is similar to that for $\mu^{P C}$ : without a carbon tax and for relatively low $F$, an infinitesimal loss in RE storage efficiency prompts a perfectly competitive $\mathrm{RE}$ producer to put more value on storage, i.e., by preserving more of the RE resource for period 2. However, a relatively inefficient storage device renders this strategy ineffective, thereby inducing more use of the RE resource in period 1 . With a carbon tax, only for $F<1.05$ does the RE producer shift its RE stock to period 1 (Figure 16). Likewise, Figures 17 and 18 trace out the analytical results of Propositions 6 and 7 for thermal production. As emphasised 

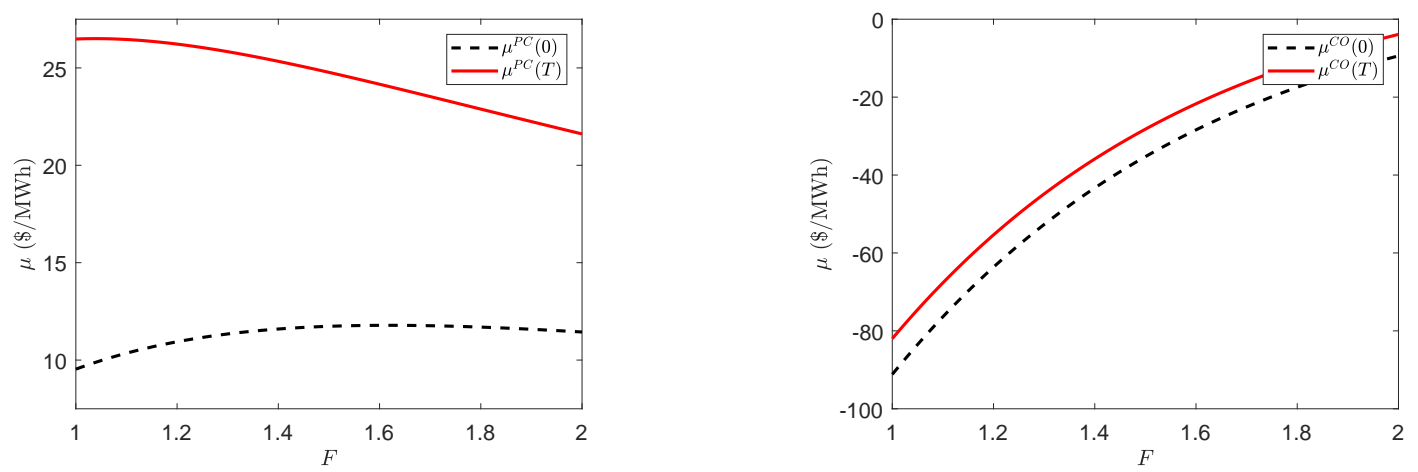

Figure 13: Impact of RE storage efficiency on RE marginalFigure 14: Impact of RE storage efficiency on RE marginal value under $\mathrm{PC}$ value under $\mathrm{CO}$

above and in Proposition 6, there is an increase in period-1 thermal production in response to an infinitesimal loss in storage efficiency for $T=0$ only if storage is relatively efficient. Indeed, a very inefficient storage device would not be worth reinforcing by shifting the RE resource from periods 1 to 2 with a countervailing increase in period-1 thermal production.
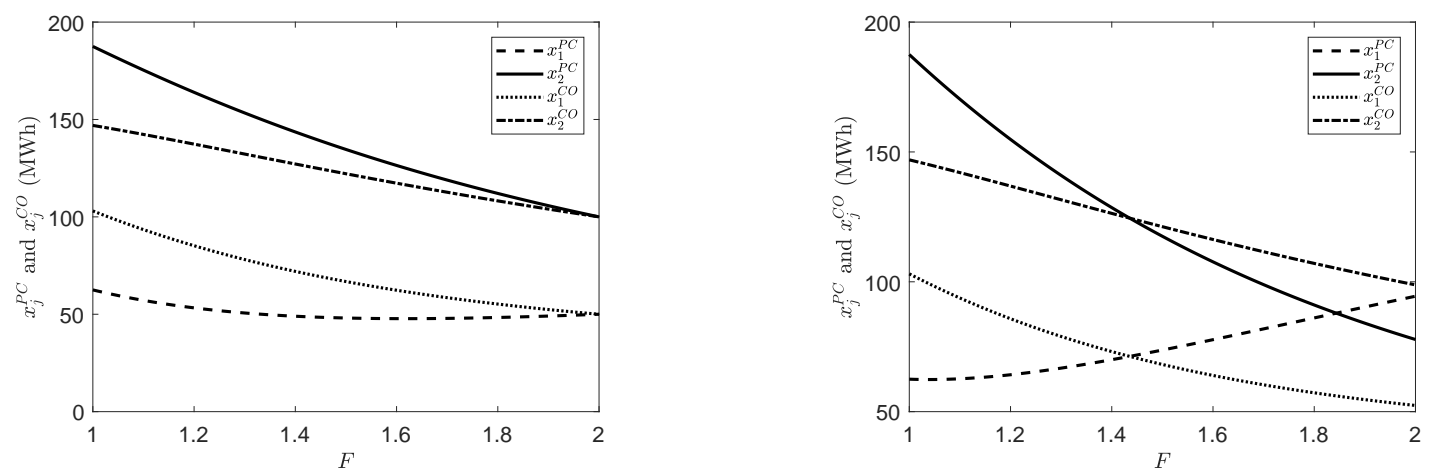

Figure 15: Impact of RE storage efficiency on RE produc-Figure 16: Impact of RE storage efficiency on RE production without a carbon tax $(T=\$ 0 / t)$ tion $(T=\$ 20 / t)$

Figures 19 and 20 reveal how producers' incentives are affected by a degradation in RE storage efficiency under carbon taxes of $\$ 0 / \mathrm{t}$ and $\$ 20 / \mathrm{t}$, respectively. As expected, the thermal producer benefits from a relatively less efficient competitor. However, the impact of $F$ on the RE producer's profit is non-monotonic regardless of the level of carbon tax: under PC, its profit actually increases with a less-efficient storage device for $F<1.618$ if $T=0(F<1.05$ if $T=20)$ as the marginal value of RE storage increases and more RE resource is preserved for the peak period. The RE producer's profit under $\mathrm{CO}$ exhibits a similar non-monotonicity as there is a "turning point" at $F=1.51$ if $T=0(F=1.36$ if $T=20)$.

Finally, Figures 21 and 22 indicate that carbon emissions decrease and social welfare increases if storage efficiency is improved, i.e., a decrease in $F$. Although these results are intuitive, they may pose a challenge for policymakers when considering the conflicting private incentives of RE producers in Figures 19 and 20. These latter two figures suggest that RE producers (whether 

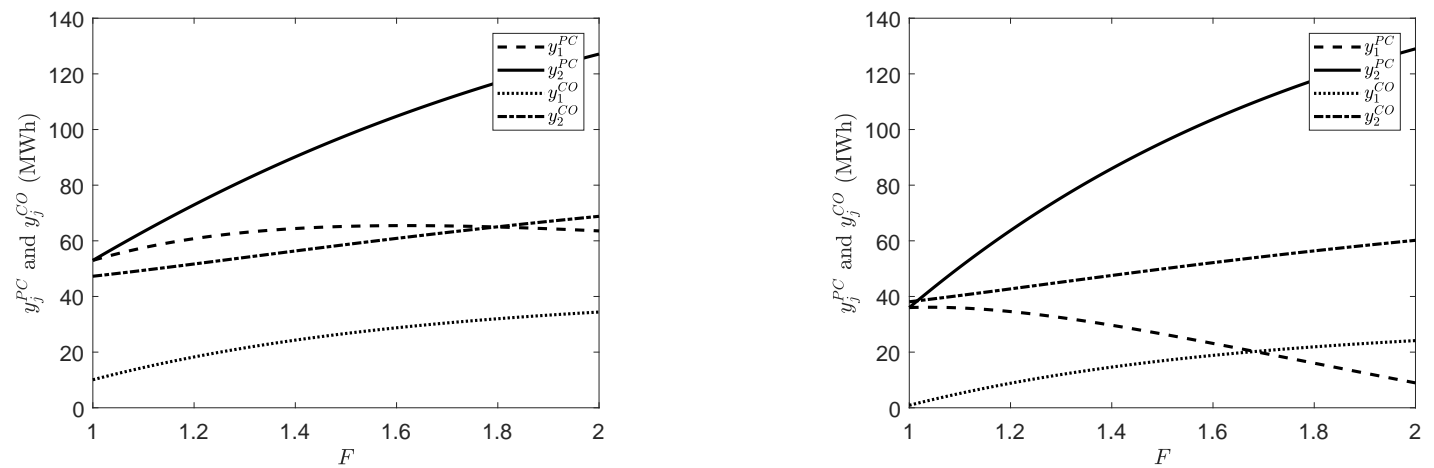

Figure 17: Impact of RE storage efficiency on thermal pro-Figure 18: Impact of RE storage efficiency on thermal production without a carbon $\operatorname{tax}(T=\$ 0 / t)$ duction $(T=\$ 20 / t)$
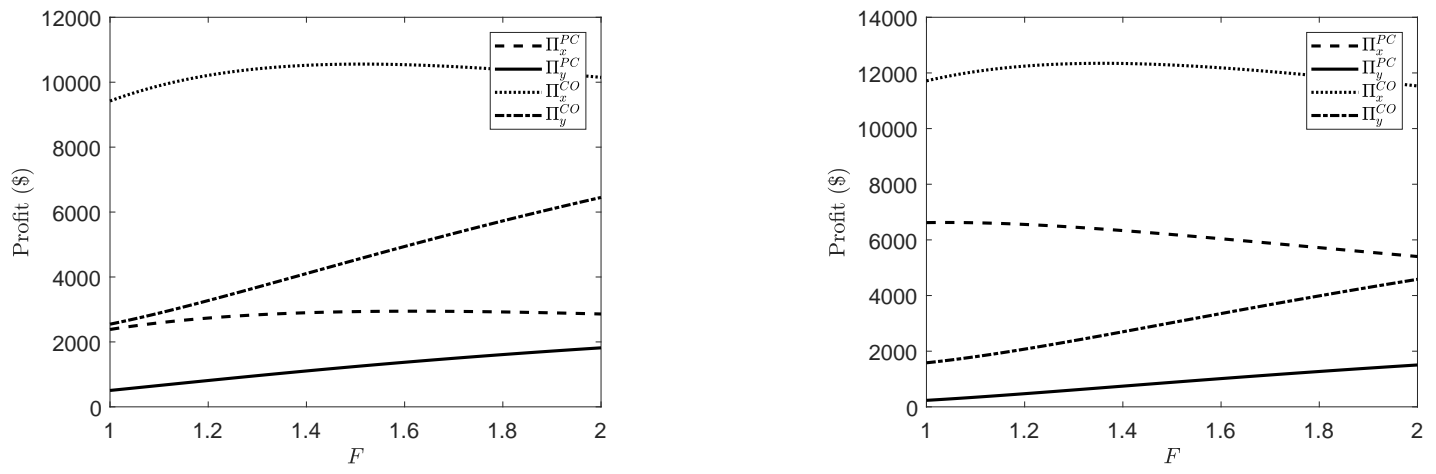

Figure 19: Impact of RE storage efficiency on profit $(T=$ Figure 20: Impact of RE storage efficiency on profit $(T=$ $\$ 0 / t)$ $\$ 20 / t)$

perfectly competitive or not) may actually be hurt by an infinitesimal increase in storage efficiency, which is related to the "turning point" discussed in the preceding two paragraphs. In this sense, carbon policy may help to align private and social incentives as the critical value of $F$ at which the "turning point" occurs is lower under a carbon tax, i.e., only a very efficient device would deter an RE producer from wanting to increase its efficiency further. This conclusion is supported by Figure 13 in which the marginal willingness to pay for more RE storage capacity increases as $F$ decreases for a wider range of $F$ when $T>0$. On the other hand, the inherent aversion to more production under $\mathrm{CO}$ as revealed in Figure 14 means that it is more challenging to align private and social incentives using carbon policy when RE producers have market power. ${ }^{7}$

\section{Conclusions}

Traditionally, electricity was treated as a non-storable product in the literature due to limited possibilities for its storage apart from hydropower reservoirs and pumped-hydro storage. However,

\footnotetext{
${ }^{7}$ We numerically investigate the robustness of the findings about the conflict between private and social incentives with respect to dynamic decisions in Appendix $\mathrm{C}$ and with respect to a quadratic emission function in Appendix D.
} 

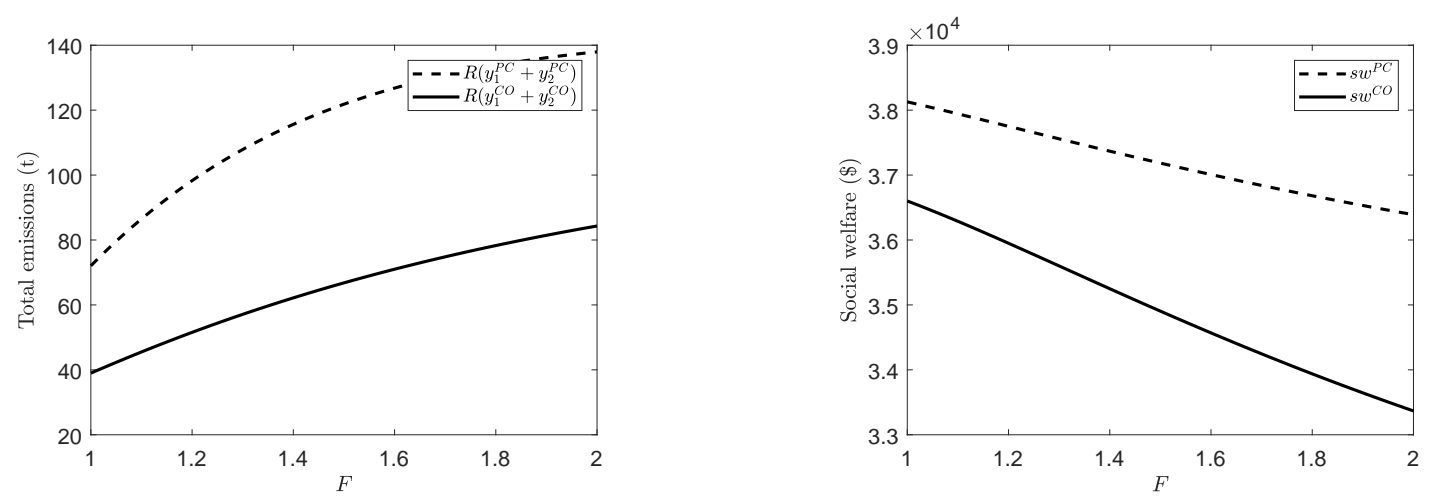

Figure 21: Impact of RE storage efficiency on carbon emis-Figure 22: Impact of RE storage efficiency on social welfare sions $(T=\$ 20 / t)$

$(T=\$ 20 / t)$

government policy in the past decade has incentivised the adoption and operation of intermittent $\mathrm{RE}$ technologies as part of its mitigation strategy against climate change. As a consequence, this development has spurred a complementary interest in energy storage, and technologies such as lithium ion, lead acid, and sodium sulfur have seen increases in installation as their capacity costs have decreased. More widespread adoption of energy storage in tandem with greater RE penetration also changes the incentives of power producers. In particular, storage may be deployed not only in a socially optimal manner but also in order to increase profits, e.g., through the exercise of market power. Indeed, the need for more flexible generation and storage engendered by the sustainableenergy transition may strengthen the position of firms that own such technologies.

In order to examine how carbon policy, RE resource availability, and storage efficiency affect producers' incentives and the value of storage, we develop a market-equilibrium model with inefficient storage. Such a framework enables us to address these aspects, and we subsequently specify the conditions under which RE producers with storage are inclined to shift deployment of the RE resource and its implications for the marginal value of RE storage. We are, thus, able to formalise the conditions under which results observed in the hydropower literature, viz., the shifting of the $\mathrm{RE}$ resource to off-peak periods and negative marginal values in the presence of market power, may not hold. Our comparative statics with respect to the carbon tax, resource availability (or storage capacity), and storage efficiency provide relevant policy and technological insights. In particular, we are able to explain why private RE storage owners may not welcome an exogenous improvement in storage efficiency and how private incentives for storage investment may be better aligned with social ones via carbon policy.

Future work in this area could directly tackle the storage-investment decision by taking a bi-level approach to investigate the RE producer's incentive to modify installed capacity in the presence of a more stringent carbon policy or higher RE storage efficiency (Siddiqui et al., 2016). An analysis in the presence of transmission constraints would also be pertinent given the extent to which transmission and storage can be considered as variation-management tools by shifting $\mathrm{RE}$ stocks both spatially and temporally in response to policy and technological changes (Sauma and Oren, 2009). Alternatively, the extent to which the findings here carry over to a more realistic 
setting, e.g., as in either Bushnell (2003) or Virasjoki et al. (2016), would also be worthwhile to explore in terms of the impacts of ownership structures and variability in RE output.

\section{Acknowledgements}

This work has been supported by funding received from the Social Sciences and Humanities Research Council (SSHRC) of Canada under grant number 435-2017-0068 and HEC Montréal's Chair in Energy Sector Management. We have benefited from feedback received at the 2018 International Conference of the International Association for Energy Economics and a seminar at the Technical University of Denmark. Comments from the handling editor and two anonymous referees have greatly improved the paper. All remaining errors are the authors' own.

\section{References}

Abada, I., S.A. Gabriel, V. Briat, and O. Massol (2013), "A Generalized Nash-Cournot Model for the Northwestern European Natural Gas Markets with a Fuel Substitution Demand Function: The GaMMES Model," Networks \& Spatial Econ. 13: 1-42.

Benchekroun, H., A. Halsema, and C. Withagen (2010), "When Additional Resource Stocks Reduce Welfare," J. Env. Econ. \& Mgmt. 59: 109-114.

Borenstein, S., J. Bushnell, and S. Stoft (2000), "The Competitive Effects of Transmission Capacity in a Deregulated Electricity Industry," RAND J. Econ. 31: 294-325.

Bulow, J.I., J.D. Geanakoplos, and P.D. Klemperer (1985), "Multimarket Oligopoly: Strategic Substitutes and Complements," J. Pol. Econ. 93(3): 488-511.

Bushnell, J. (2003), "A Mixed Complementarity Model of Hydrothermal Electricity Competition in the Western United States," Oper. Res. 51: 80-93.

California ISO (2016), What the Duck Curve Tells Us About Managing a Green Grid, https:// www.caiso.com/documents/flexibleresourceshelprenewables_fastfacts.pdf, Folsom, CA, USA.

California Legislative Information (2015), SB-350 Clean Energy and Pollution Reduction Act of 2015, http://leginfo.legislature.ca.gov/faces/billNavClient.xhtml?bill_id= 201520160SB350, Sacramento, CA, USA.

Crampes, C. and M. Moreaux (2001), "Water Resource and Power Generation," Intl. J. Ind. Org. 19: $975-997$.

Downward, A., G. Zakeri, and A.B. Philpott (2010), "On Cournot Equilibria in Electricity Transmission Networks," Oper. Res. 58(4): 1194-1209. 
European Commission (2009), Directive 2009/28/EC of the European Parliament and of the Council, http://eur-lex . europa.eu/legal-content/EN/TXT/?uri=CELEX : 32009L0028, Brussels, Belgium.

Førsund, F.R. (2015), Hydropower Economics, Springer International, New York, NY, USA.

Gabriel, S.A., A.J. Conejo, J.D. Fuller, B.F. Hobbs, and C. Ruiz (2013), Complementarity Modeling in Energy Markets, Springer International, New York, NY, USA.

Global CCS Institute (2011), Bridging the Commercial Gap for Carbon Capture and Storage: ERCOT Market Analysis, https://hub.globalccsinstitute.com/publications/ bridging-commercial-gap-carbon-capture-and-storage/40-ercot-market-analysis, Melbourne, Australia.

Kunz, F. (2013), "Improving Congestion Management: How to Facilitate the Integration of Renewable Generation in Germany," The Energy J. 34: 55-78.

Mathiesen, L., J. Skaar, and L. Skørgard (2013), "Electricity Production in a Hydro System with a Reservoir Constraint," Scand. J. Econ. 115: 575-594.

Rebennack, S., B. Flach, M.V.F. Pereira, and P.M. Pardalos (2012), "Stochastic Hydro-Thermal Scheduling Under $\mathrm{CO}_{2}$ Emissions Constraints," IEEE Trans. on Power Syst. 27(1): 58-68.

Rintamäki, T., A.S. Siddiqui, and A. Salo (2017), "Does Renewable Energy Generation Decrease the Volatility of Electricity Prices? An Analysis of Denmark and Germany," Energy Econ. 62: 270-282.

Sauma, E.E. and S.S. Oren (2009), "Do Generation Firms in Restructured Electricity Markets Have Incentives to Support Social-Welfare-Improving Transmission Investments?" Energy Econ. 31: $676-689$.

Shahmohammadi, A., R. Sioshansi, A.J. Conejo, and A. Afsharnia (2018), "Market Equilibria and Interactions Between Strategic Generation, Wind, and Storage," Applied Energy, forthcoming.

Siddiqui, A.S., M. Tanaka, and Y. Chen (2016), "Are Targets for Renewable Portfolio Standards Too Low? The Impact of Market Structure on Energy Policy," Eur. J. Oper. Res. 250(1): 328-341.

Sioshansi, R. (2010), "Welfare Impacts of Electricity Storage and the Implications of Ownership Structure," The Energy J. 31: 173-198.

Sioshansi, R. (2014), "When Energy Storage Reduces Social Welfare," Energy Econ. 41: 106-116.

Steeger G., L.A. Barroso, and S. Rebennack (2014), "Optimal Bidding Strategies for Hydro-Electric Producers: A Literature Survey," IEEE Trans. on Power Syst. 29(4): 1758-1766.

Timera Energy (2013), Gas Plant 8 Renewable Penetration: a U.K. Case Study, https:// timera-energy.com/gas-plant-renewable-penetration-a-uk-case-study/, London, U.K. 
Virasjoki, V., P. Rocha, A.S. Siddiqui, and A. Salo (2016), "Market Impacts of Energy Storage in a Transmission-Constrained Power System," IEEE Trans. on Power Syst. 31: 4108-4117.

von Hirschhausen, C. (2014), "The German 'Energiewende'-An Introduction," Econ. Energy Env. Pol. 3: 1-12.

\section{Appendix A. Equilibrium Characterisation}

As in Borenstein et al. (2000), Crampes and Moreaux (2001), and related work, we restrict our analysis to interior solutions with $\mu^{C O}<0$ as in Bushnell (2003) defined as follows:

Definition 1. The interior solution set for perfect competition is defined as

$$
\mathcal{S}^{P C}=\left\{(\mathbf{x}, \mathbf{y})^{P C} \in \mathbb{R}_{+}^{4} \mid x_{j}^{P C}>0, y_{j}^{P C}>0, j=1,2\right\}
$$

where $(\mathbf{x}, \mathbf{y})^{P C}=\left(x_{1}^{P C}, x_{2}^{P C}, y_{1}^{P C}, y_{2}^{P C}\right)$.

Definition 2. The interior solution set for Cournot oligopoly with $\mu<0$ is defined as

$$
\mathcal{S}_{\mu}^{C O}=\left\{(\mathbf{x}, \mathbf{y})_{\mu}^{C O} \in \mathbb{R}_{+}^{4} \mid \mu^{C O}<0, x_{j}^{C O}>0, y_{j}^{C O}>0, j=1,2\right\}
$$

An interior solution is ensured only if parameters are contained within some interval. This calibration is the objective of the following lemma ${ }^{8}$ for $\mathrm{PC}$ :

Lemma 1. $\mathcal{S}^{P C}$ is non-empty if and only if

$$
\begin{aligned}
& \text { (i) } D>F\left(A_{2}-F A_{1}-(F-1) \frac{R T}{C}\right) \text { if } R T \leq \frac{C\left(A_{2}-F A_{1}\right)}{F-1} \\
& \text { (ii) } D>\frac{-A_{2}+F A_{1}+(F-1) R T / C}{F} \text { if } R T>\frac{C\left(A_{2}-F A_{1}\right)}{F-1} \\
& \text { (iii) } D<A_{1}+F A_{2}-F(F-1) \frac{R T}{C}-\left(1+F^{2}\right) R T \\
& \text { (iv) } R T<\min \left\{A_{1}, \frac{A_{2}}{F+\frac{F-1}{C}}\right\} .
\end{aligned}
$$

Furthermore, $\mu^{P C}>0$ is in $\mathcal{S}^{P C}$.

Figures A.23-A.24 display all of the necessary information to characterise $\mathcal{S}^{P C}$ with respect to $D$ and $R T$. The dashed lines represent the bounds for which each variable is interior, marked by the side on which the variable is written. The binding conditions described in Lemma 1 are put between parentheses on the corresponding cutting planes. Note that this set shrinks considerably depending on the relative size of $A_{2}$ with respect to $A_{1}$.

\footnotetext{
${ }^{8}$ Proofs of all lemmata and propositions are in Appendix B.
} 


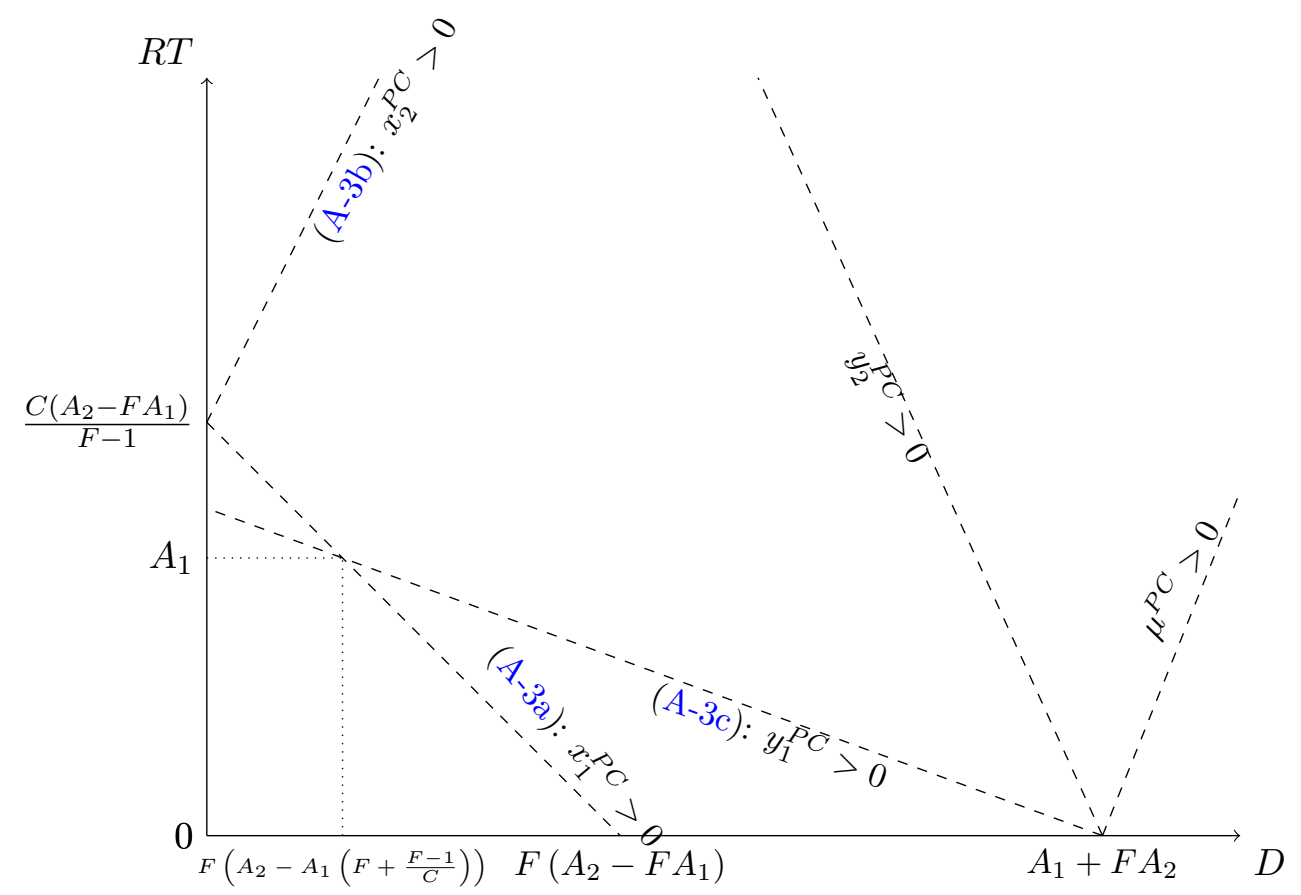

Figure A.23: Interior solution set under perfect competition if $A_{2}>A_{1}\left(F+\frac{F-1}{C}\right)$

Similar to Lemma 1, the following one provides conditions on parameters to ensure interior solutions under $\mathrm{CO}$ with $\mu^{C O}<0$ :

Lemma 2. $\mathcal{S}_{\mu}^{C O}$ is non-empty if and only if

(i) $D>\frac{(C+1)\left(A_{1}+F A_{2}\right)+R T(1+F)}{2 C+3}$

(ii) $D<\frac{A_{1}(C+2)\left(1+F^{2}\right)+\left(A_{1}+F A_{2}\right)(C+1)-R T\left[(2 C+3)\left(1+F^{2}\right)+F(F-1)\right]}{2 C+3}$

(iii) $R T<\frac{A_{1}}{2}$

A sketch of the interior solution set for $\mathrm{CO}$ is indicated in Figure A.25.

Given the characterisation of the $\mathrm{PC}$ and $\mathrm{CO}$ interior solution sets, their intersection is defined as $\mathcal{S} \equiv \mathcal{S}^{P C} \cap \mathcal{S}_{\mu}^{C O}$. This leads to the following result about the conditions for the existence of $\mathcal{S}$ : 


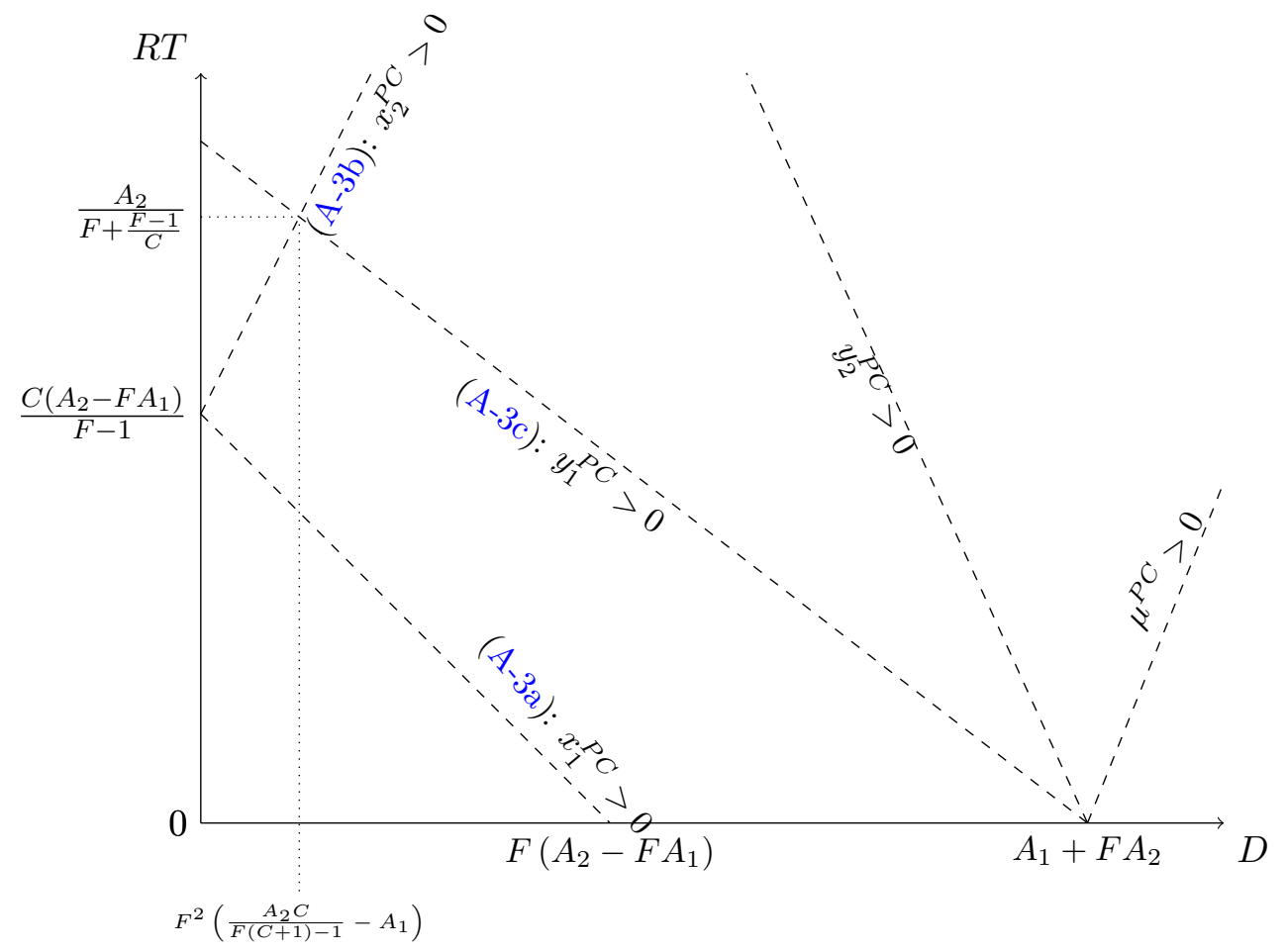

Figure A.24: Interior solution set under perfect competition if $A_{2}<A_{1}\left(F+\frac{F-1}{C}\right)$

Lemma 3. The intersection of the two interior solution sets, $\mathcal{S}$, is defined such that

(i) The lower bound on $D$ is:

$\underline{D} \equiv \begin{cases}(\mathrm{A}-4 \mathrm{a}) & \text { if }\left\{\begin{array}{l}\frac{A_{2}}{A_{1}}<F+\frac{\left(1+F^{2}\right)(C+1)}{F(C+2)} \text { for any } R T<\frac{A_{1}}{2} \\ \frac{A_{2}}{A_{1}} \in\left[F+\frac{\left(1+F^{2}\right)(C+1)}{F(C+2)}, \frac{2 C+3}{C+2}\left(F+\frac{1}{2 F}\right)+\frac{F-1+C}{2 C(C+2)}\right] \text { for } R T \in\left[\gamma, \frac{A_{1}}{2}\right)\end{array}\right. \\ (\mathrm{A}-3 \mathrm{a}) & \text { if } \frac{A_{2}}{A_{1}}>\frac{2 C+3}{C+2}\left(F+\frac{1}{2 F}\right)+\frac{F-1+C}{2 C(C+2)} \text { and } R T<\min \left\{\gamma, \delta^{+}\right\}\end{cases}$

(ii) The upper bound on $D$ is:

$\bar{D} \equiv\left\{\begin{array}{c}(\mathrm{A}-4 \mathrm{~b}) \quad \text { if }\left\{\begin{array}{l}\frac{A_{2}}{A_{1}} \geq F+\frac{(F-1)(C+3)}{2 C(C+2)} \text { for any } R T<\frac{A_{1}}{2} \\ \frac{A_{2}}{A_{1}}<F+\frac{(F-1)(C+3)}{2 C(C+2)} \text { for } R T \leq \alpha\end{array}\right. \\ (\mathrm{A}-3 \mathrm{c}) \quad \text { otherwise and } R T<\beta\end{array}\right.$ 


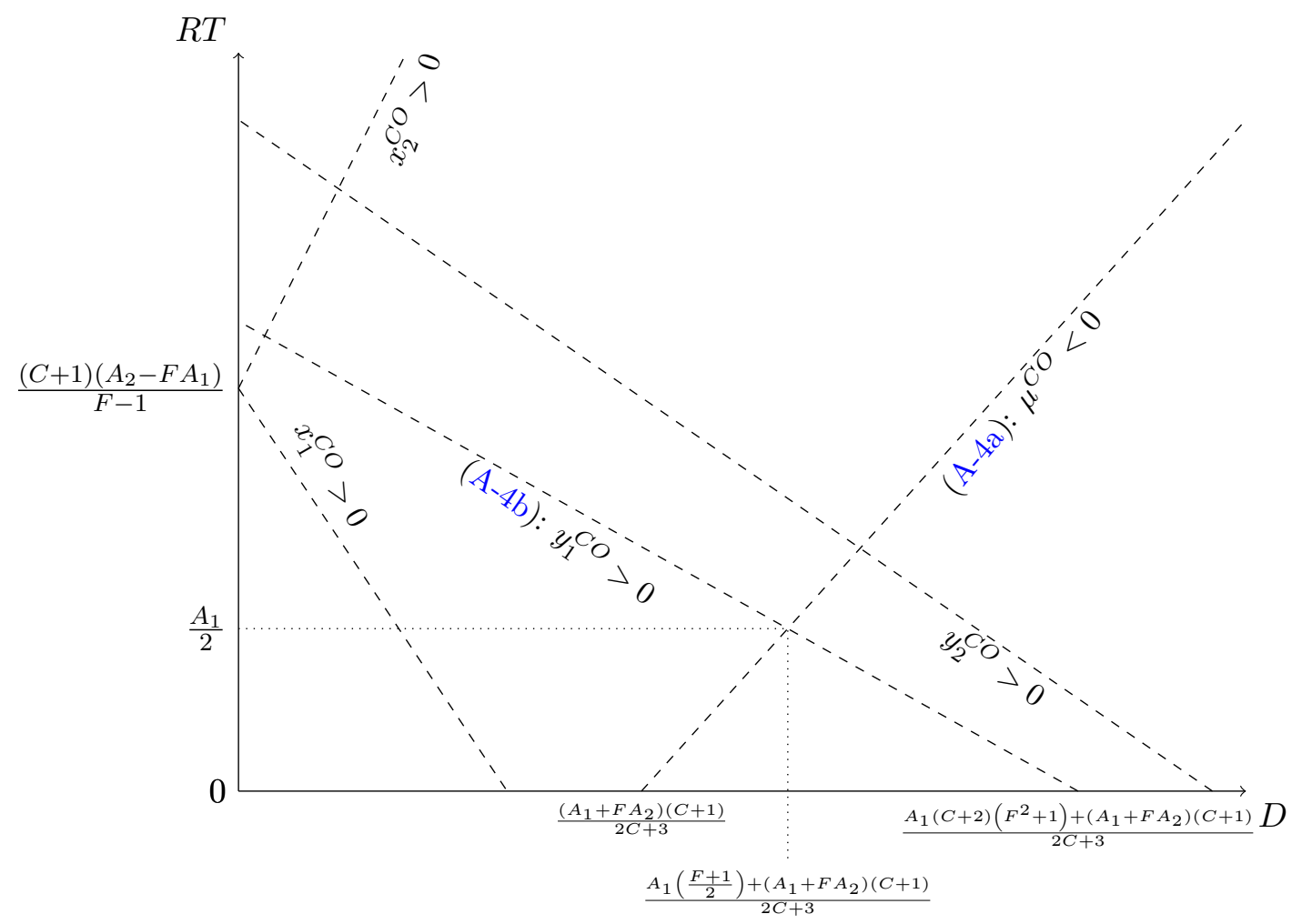

Figure A.25: Interior solution set under Cournot oligopoly

where

$$
\begin{aligned}
\alpha & =\frac{(C+2)\left(A_{2}-F A_{1}\right)}{(F-1)\left(1+\frac{3}{C}\right)} \\
\beta & =\frac{(C+2)\left(A_{1}+F A_{2}\right)}{2(C+2)\left(1+F^{2}\right)+F(F-1)\left(1+\frac{3}{C}\right)} \\
\gamma & =\frac{F(C+2)\left(A_{2}-F A_{1}\right)-A_{1}\left(1+F^{2}\right)(C+1)}{1+F\left[1+\frac{F-1}{C}(2 C+3)\right]} \\
\delta^{+} & =\frac{A_{1}(2 C+3)\left(1+F^{2}\right)-F(C+2)\left(A_{2}-F A_{1}\right)}{(2 C+3)\left(1+F^{2}\right)-F(F-1)\left(1+\frac{3}{C}\right)}>0, \text { or does not apply. }
\end{aligned}
$$

Note that in Lemma $3, \frac{A_{1}}{2}, \alpha, \beta, \gamma$, and $\delta^{+}$are the potential vertices of $\mathcal{S}$ for $T>0$, i.e., ${ }^{9}$

- $R T=\frac{A_{1}}{2}$ is where the interior constraints on $y_{1}^{C O}>0$ and $\mu^{C O}<0$ cross,

- $R T=\alpha$ is for the crossing of $y_{1}^{C O}>0$ and $y_{1}^{P C}>0$,

- $R T=\beta$ is for the crossing of $y_{1}^{P C}>0$ and $\mu^{C O}<0$,

\footnotetext{
${ }^{9}$ In addition, the crossing of the interior constraints on $x_{1}^{P C}>0$ and $y_{1}^{P C}>0$ is for $R T=A_{1}$, which is always outside $\mathcal{S}$ since $\mathcal{S}_{\mu}^{C O}$ is defined only for $R T<\frac{A_{1}}{2}$. Furthermore, the crossing of the interior constraints on $x_{2}^{P C}>0$ and $\mu^{C O}<0$ is outside $\mathcal{S}$ for $C>3-2 \sqrt{2}$.
} 
- $R T=\gamma$ is for the crossing of $x_{1}^{P C}>0$ and $\mu^{C O}<0$, and

- $R T=\delta^{+}$is for the crossing of $x_{1}^{P C}>0$ and $y_{1}^{C O}>0$.

\section{Appendix B. Proofs of Lemmata and Propositions}

\section{Proof of Lemma 1}

1. $y_{j}^{P C}>0 \forall j \Longleftrightarrow D<A_{1}+F A_{2}-F(F-1) R T / C-\left(1+F^{2}\right) R T$, thereby yielding (A-3c) as in part (iii) of Lemma 1.

(a) $y_{1}^{P C}>0 \Longleftrightarrow D<A_{1}+F A_{2}-F(F-1) R T / C-\left(1+F^{2}\right) R T$ from (15).

(b) $y_{2}^{P C}>0 \Longleftrightarrow D<A_{1}+F A_{2}+F(F-1) R T / F C-\left(1+F^{2}\right) R T / F$ from (16).

(c) Comparing the two, $y_{1}^{P C}>0 \Longrightarrow y_{2}^{P C}>0$ because $\left(1+F^{2}\right)(1 / F-1) \leq 0 \leq(F-$ 1) $(1 / F+F)$.

2. The conditions for $y_{j}^{P C}>0 \forall j$ imply that $\mu^{P C}>0$ from (17).

3. $x_{j}^{P C}>0 \Longleftrightarrow D>\max \left\{F\left(A_{2}-F A_{1}-(F-1) R T / C\right),\left(-A_{2}+F A_{1}+(F-1) R T / C\right) / F\right\}$, $\forall j$, thereby yielding (A-3a) or (A-3b) accordingly. These correspond to parts (i) and (ii) of Lemma 1. The first term is greater for $R T \leq C\left(A_{2}-F A_{1}\right) /(F-1)$ :

(a) $x_{1}^{P C}>0 \Longleftrightarrow D>F\left(A_{2}-F A_{1}-(F-1) R T / C\right)$ in (13),

(b) $x_{2}^{P C}>0$ for any $D>\left(-A_{2}+F A_{1}+(F-1) R T / C\right) / F$ in (14).

(c) Comparing the two, $F\left(A_{2}-F A_{1}-(F-1) R T / C\right) \geq\left(-A_{2}+F A_{1}+(F-1) R T / C\right) / F$, and, thus, $x_{1}^{P C}>0 \Longrightarrow x_{2}^{P C}>0 \Longleftrightarrow R T \leq C\left(A_{2}-F A_{1}\right) /(F-1)$, and vice versa.

4. Comparing the upper bound in each case, the interval is non-empty only

$$
\begin{cases}\text { if } A_{2}>A_{1}\left(F+\frac{F-1}{C}\right) & \text { and } R T<A_{1} \text {, or } \\ \text { if } A_{2}<A_{1}\left(F+\frac{F-1}{C}\right) & \text { and } R T<\frac{A_{2}}{F+\frac{F-1}{C}},\end{cases}
$$

thereby yielding (A-3d) in part (iv) of Lemma 1 since $R T$ has to be less than both $A_{1}$ and $\frac{A_{2}}{F+\frac{F-1}{C}}$.

(a) If $R T \leq C\left(A_{2}-F A_{1}\right) /(F-1)$, then we compare Case 1 with Case 3(a), i.e., ensure that the upper bound on $D$ defined by (A-3c) is greater than the lower bound on $D$ defined by (A-3a). The interval defined by these two bounds is non-empty if $F\left(A_{2}-F A_{1}-(F-1) R T / C\right)<A_{1}+F A_{2}-F(F-1) R T / C-\left(1+F^{2}\right) R T$, which leads to $R T<A_{1}$. This bound is binding if $A_{1}<C\left(A_{2}-F A_{1}\right) /(F-1)$, i.e., if $\frac{A_{2}}{A_{1}}>F+\frac{F-1}{C}$. 
(b) If $R T>C\left(A_{2}-F A_{1}\right) /(F-1)$, then we compare Case 1 with Case 3(b), i.e., ensure that the upper bound on $D$ defined by (A-3c) is greater than the lower bound on $D$ defined by (A-3b). The interval is non-empty if $\left(-A_{2}+F A_{1}+(F-1) R T / C\right) / F<A_{1}+F A_{2}-$ $F(F-1) R T / C-\left(1+F^{2}\right) R T$, which leads to $R T<\frac{A_{2}}{F+\frac{F-1}{C}}$. For this case to be feasible, i.e., for $\frac{A_{2}}{F+\frac{F-1}{C}}>\frac{C\left(A_{2}-F A_{1}\right)}{F-1}$, it must be the case that $A_{2}>\frac{C\left(A_{2}-F A_{1}\right)}{F-1}\left(F+\frac{F-1}{C}\right)$, i.e., if $\frac{A_{2}}{A_{1}}<F+\frac{F-1}{C}$.

\section{Proof of Lemma 2}

1. $\mu^{C O}<0 \Longleftrightarrow D>\frac{(C+1)\left(A_{1}+F A_{2}\right)+R T(1+F)}{2 C+3}$ from (22), thereby yielding (A-4a).

2. $\mu^{C O}<0 \Longrightarrow x_{j}^{C O}>0 \forall j$. This is easily verifiable by comparing (22) with (18)-(19).

3. $y_{j}^{C O}>0 \forall j \Longleftrightarrow D<\frac{A_{1}(C+2)\left(1+F^{2}\right)+\left(A_{1}+F A_{2}\right)(C+1)-R T\left[(2 C+3)\left(1+F^{2}\right)+F(F-1)\right]}{2 C+3}$, thereby yielding $(\mathrm{A}-4 \mathrm{~b})$ :

(a) $y_{1}^{C O}>0$ if and only if from $(20) D<\frac{A_{1}(C+2)\left(1+F^{2}\right)+\left(A_{1}+F A_{2}\right)(C+1)-R T\left[(2 C+3)\left(1+F^{2}\right)+F(F-1)\right]}{2 C+3}$.

(b) $y_{2}^{C O}>0$ if and only if from (21) $D<\frac{A_{2}(C+2)\left(1+F^{2}\right)+F\left(A_{1}+F A_{2}\right)(C+1)-R T\left[(2 C+3)\left(1+F^{2}\right)+1-F\right]}{F(2 C+3)}$.

(c) Comparing the two, $(C+2)\left(1+F^{2}\right)\left(F A_{1}-A_{2}-2(F-1) R T\right)<0$, and, hence, $y_{1}^{C O}>$ $0 \Longrightarrow y_{2}^{C O}>0$.

4. The two bounds on $D$ define a non-empty set only if $F\left[(C+1)\left(A_{1}+F A_{2}\right)+R T(1+F)\right]<$ $A_{2}(C+2)\left(1+F^{2}\right)+F\left(A_{1}+F A_{2}\right)(C+1)-R T\left[(2 C+3)\left(1+F^{2}\right)+1-F\right]$, i.e., if $R T<A_{1} / 2$, thereby yielding $(\mathrm{A}-4 \mathrm{c})$.

Proof of Lemma 3 We identify conditions under which $\mathcal{S}_{\mu}^{C O}$ is contained in $\mathcal{S}^{P C}$ by pairwise comparison of the bounds for interior solutions in both PC and CO cases. First, we compare lower bounds between themselves to characterise the conditions for which one is binding for $\mathcal{S}$. Next, we complete the characterisation of $\mathcal{S}$ by comparing each lower bound with each upper bound in order to define the potential vertices of $\mathcal{S}$.

A. Checking for the lower bound on $D$ with respect to $\mu^{C O}<0$ and $x_{1}^{P C}>0$, i.e., comparing $(\mathrm{A}-4 \mathrm{a})$ and $(\mathrm{A}-3 \mathrm{a})$ :

(a) $\mathcal{S}_{\mu}^{C O}$ is more binding than $\mathcal{S}^{P C}$, i.e., the right-hand side of $(\mathrm{A}-4 \mathrm{a})$ is lower than the right-hand side of (A-3a), if and only if $R T>\frac{F(C+2)\left(A_{2}-F A_{1}\right)-A_{1}\left(1+F^{2}\right)(C+1)}{1+F^{2}+F(F-1)\left(1+\frac{3}{C}\right)} \equiv \gamma$.

(b) The threshold $\gamma$ is lower than zero, i.e., $\mathcal{S}_{\mu}^{C O}$ is always more binding than $\mathcal{S}^{P C}$, if $\frac{A_{2}}{A_{1}}<$ $F+\frac{\left(1+F^{2}\right)(C+1)}{F(C+2)}$. 
(c) The threshold $\gamma$ is higher than $\frac{A_{1}}{2}$, i.e., $\mathcal{S}_{\mu}^{C O}$ is never more binding than $\mathcal{S}^{P C}$, if

$$
\frac{A_{2}}{A_{1}}>F+\frac{2 C+3+2 F^{2}(C+1)+F\left(\frac{F-1}{C}+1\right)}{2 F(C+2)},
$$

thereby yielding the first case for $\underline{D}$ in (A-5a).

A'. Checking for the lower bound on $D$ with respect to $\mu^{C O}<0$ and $x_{2}^{P C}>0$, i.e., comparing $(\mathrm{A}-4 \mathrm{a})$ and $(\mathrm{A}-3 \mathrm{~b})$ :

(a) $\mathcal{S}_{\mu}^{C O}$ is more binding than $\mathcal{S}^{P C}$, i.e., the right-hand side of $(\mathrm{A}-4 \mathrm{a})$ is lower than the right-hand side of $(\mathrm{A}-3 \mathrm{~b})$, if and only if

$$
(C+2)\left(A_{2}-F A_{1}\right)+A_{2}\left(1+F^{2}\right)(C+1)>R T\left[\frac{F-1}{C}(2 C+3)-F(1+F)\right] .
$$

While the left-hand side of (B-2) is strictly positive, the right-hand side may be nonpositive. In this case, $\mathcal{S}_{\mu}^{C O}$ is always more binding than $\mathcal{S}^{P C}$.

(b) Assuming that the right-hand side is positive, we obtain an upper bound on $R T$ under which $\mathcal{S}_{\mu}^{C O}$ is always more binding than $\mathcal{S}^{P C}$. We compare the obtained upper bound on $R T$ with the upper bound in $\mathcal{S}_{\mu}^{C O}$, i.e., $\mathcal{S}_{\mu}^{C O}$ is more binding than $\mathcal{S}^{P C}$ if $\frac{A_{1}}{2}<$ $\frac{(C+2)\left(A_{2}-F A_{1}\right)+A_{2}\left(1+F^{2}\right)(C+1)}{\frac{(F-1)}{C}(2 C+3)-F(1+F)}$.

(c) Rearranging the latter expression, we obtain a ratio $\frac{A_{2}}{A_{1}}>\frac{(2 C+3)\left(F+\frac{F-1}{C}\right)-F^{2}}{2\left[2 C+3+F^{2}(C+1)\right]}$. If this threshold is not greater than $F$, then this bound is not binding such that $\mathcal{S}_{\mu}^{C O}$ is more binding than $\mathcal{S}^{P C}$, i.e.,

$$
\frac{(2 C+3)\left(F+\frac{F-1}{C}\right)-F^{2}}{2\left[2 C+3+F^{2}(C+1)\right]} \leq F \Longleftrightarrow F^{2} C-F(1-C)+1 \geq 0 .
$$

It is immediate that for any $C \geq 1$, this polynomial is positive. The corresponding discriminant with respect to $F$ of this polynomial is:

$$
\Delta_{F}=C^{2}-6 C+1 \leq 0 \Longleftrightarrow C \in[3-2 \sqrt{2}, 3+2 \sqrt{2}]
$$

(d) Since (B-3) describes a quadratic convex function, it has a unique minimum, which is equal to zero for the bounds described in (B-4), and $\lim _{F \rightarrow+\infty}=+\infty$ due to the positive term on $F^{2}$ in (B-3). Hence, if (B-3) does not change sign, then it must be positive.

(e) If $C=3+2 \sqrt{2}>1$, then (B-3) $>0$ for any $F$.

(f) For the lower bound on $C$, the minimum at zero is attained for $F=(\sqrt{2}-1) /(3-2 \sqrt{2})$. For any $C$ above this threshold, the sign of (B-3) does not vary and must be positive for any $F$ since its limit in $F$ is positive. Below this lower bound, (B-3) has two roots, which implies that is negative for some $F$. 
(g) Hence, the assumption $C>3-2 \sqrt{2}$ is necessary and sufficient to ensure that $\mu^{C O}<$ $0 \Longrightarrow x_{2}^{P C}>0$ for all $F>1$.

B. Checking for the condition on the upper bound of $D$ with respect to $y_{1}^{P C}>0$ and $y_{1}^{C O}$, i.e., comparing (A-3c) and (A-4b):

(a) $\mathcal{S}_{\mu}^{C O}$ is more binding than $\mathcal{S}^{P C}$, i.e., the right-hand side of (A-3c) is greater than that of (A-4b), for any $R T<\frac{(C+2)\left(A_{2}-F A_{1}\right)}{(F-1)\left(1+\frac{3}{C}\right)} \equiv \alpha$. Provided that $\mathcal{S}_{\mu}^{C O}$ is defined such that $R T \frac{<A_{1}}{2}$, this implies the following bounds on $\frac{A_{2}}{A_{1}}$ :

$$
\frac{A_{1}}{2}<\frac{(C+2)\left(A_{2}-F A_{1}\right)}{(F-1)\left(1+\frac{3}{C}\right)} \Longleftrightarrow \frac{A_{2}}{A_{1}}>F+\frac{(F-1)(C+3)}{2 C(C+2)}
$$

thereby yielding the first case for $\bar{D}$ in (A-5b).

(b) Otherwise, (A-3c) is the binding bound.

Finally, we complete the characterisation of $\mathcal{S}$ by checking the upper bounds on $R T$, i.e., assessing under which conditions on $R T$ the lower and upper bounds cross each other:

1. Checking whether the constraints $y_{1}^{P C}>0$ and $x_{1}^{P C}>0$ may constitute a vertex of $\mathcal{S}$, i.e., comparing (A-3c) with (A-3a), in $\mathcal{S}$. From Lemma 1, the right-hand side of (A-3a) crosses with that of (A-3c) for $R T=A_{1}$, which is outside $\mathcal{S}_{\mu}^{C O}$ as defined in Lemma 2. Hence, such a crossing is not defined within $\mathcal{S}$.

2. Checking whether the constraints $y_{1}^{P C}>0$ and $\mu^{C O}<0$ may constitute a vertex of $\mathcal{S}$, i.e., comparing (A-3c) with (A-4a), in $\mathcal{S}$. Such a crossing occurs at $R T=\frac{(C+2)\left(A_{1}+F A_{2}\right)}{2(C+2)\left(F^{2}+1\right)+F(F-1)\left(1+\frac{3}{C}\right)} \equiv$ $\beta$. For this crossing to be a vertex of $\mathcal{S}$, it must take place when $y_{1}^{P C}>0$ is the binding condition defining $\mathcal{S}$, i.e., $\beta<\frac{A_{1}}{2}$. This implies that $\frac{A_{2}}{A_{1}}<F+\frac{(F-1)\left(1+\frac{3}{C}\right)}{2(C+2)}$, thereby yielding the second case for $\bar{D}$ in $(\mathrm{A}-5 \mathrm{~b})$.

3. Checking whether the constraints $y_{1}^{C O}>0$ and $x_{1}^{P C}>0$ may constitute a vertex of $\mathcal{S}$, i.e., comparing (A-4b) with (A-3a), in $\mathcal{S}$. Such a crossing occurs at $R T=\frac{A_{1}(2 C+3)\left(1+F^{2}\right)-F(C+2)\left(A_{2}-F A_{1}\right)}{(2 C+3)\left(1+F^{2}\right)-F(F-1)\left(1+\frac{3}{C}\right)} \equiv$ $\delta$. In order for this crossing to be in $\mathcal{S}$, it must take place when $x_{1}^{P C}>0$ is the binding condition defining $\mathcal{S}$, i.e., $\delta<\gamma$. This implies that

$$
\frac{A_{2}}{A_{1}}>F+\frac{(2 C+3)\left(F^{2}+1\right)+F(F-1)\left(1+\frac{3}{C}\right)}{2 F(C+2)}
$$

The lower bound in (B-5) is implied by (B-1), thereby yielding the second case for $\underline{D}$ in (A-5a).

Proof of Proposition 1 The results and signs are evident upon partial differentiation of the 
interior solutions for PC in $\mathcal{S}^{P C}, x_{j}^{P C}, y_{j}^{P C}, j=1,2, \mu^{P C}$, in (13)-(17) with respect to $T$ :

$$
\begin{aligned}
\frac{\partial x_{1}^{P C}}{\partial T} & =\frac{F(F-1) R}{C\left(1+F^{2}\right)}>0 \\
\frac{\partial x_{2}^{P C}}{\partial T} & =\frac{-(F-1) R}{C\left(1+F^{2}\right)}<0 \\
\frac{\partial y_{1}^{P C}}{\partial T} & =-\frac{R}{(C+1)}\left[1+\frac{F(F-1)}{C\left(1+F^{2}\right)}\right]<0 \\
\frac{\partial y_{2}^{P C}}{\partial T} & =-\frac{R}{(C+1)}\left[1-\frac{(F-1)}{C\left(1+F^{2}\right)}\right]>0 \text { if } C<\frac{F-1}{1+F^{2}} \\
\frac{\partial \mu^{P C}}{\partial T} & =\frac{(F+1) R}{(C+1)\left(1+F^{2}\right)}>0
\end{aligned}
$$

Proof of Proposition 2 The results and signs are evident upon partial differentiation of the interior solutions for $\mathrm{CO}$ in $\mathcal{S}_{\mu}^{C O}, x_{j}^{C O}, y_{j}^{C O}, j=1,2, \mu^{C O}$, in (18)-(22) with respect to $T$ :

$$
\begin{aligned}
\frac{\partial x_{1}^{C O}}{\partial T} & =\frac{F(F-1) R}{(2 C+3)\left(1+F^{2}\right)}>0 \\
\frac{\partial x_{2}^{C O}}{\partial T} & =\frac{-(F-1) R}{(2 C+3)\left(1+F^{2}\right)}<0 \\
\frac{\partial y_{1}^{C O}}{\partial T} & =-\frac{R}{(C+2)}\left[1+\frac{F(F-1)}{(2 C+3)\left(1+F^{2}\right)}\right]<0 \\
\frac{\partial y_{2}^{C O}}{\partial T} & =-\frac{R}{(C+2)}\left[1-\frac{(F-1)}{(2 C+3)\left(1+F^{2}\right)}\right]<0 \\
\frac{\partial \mu^{C O}}{\partial T} & =\frac{(F+1) R}{(C+2)\left(1+F^{2}\right)}>0
\end{aligned}
$$

Proof of Proposition 3 The result follows from comparison of (18) and (13).

Proof of Proposition 4 The results and signs are evident upon partial differentiation of the interior solutions for PC in $\mathcal{S}^{P C}, x_{j}^{P C}, y_{j}^{P C}, j=1,2, \mu^{P C}$, in (13)-(17) with respect to $D$ :

$$
\begin{aligned}
\frac{\partial x_{1}^{P C}}{\partial D} & =\frac{1}{1+F^{2}}>0 \\
\frac{\partial x_{2}^{P C}}{\partial D} & =\frac{F}{1+F^{2}}>0 \\
\frac{\partial y_{1}^{P C}}{\partial D} & =-\frac{1}{(C+1)\left(1+F^{2}\right)}<0 \\
\frac{\partial y_{2}^{P C}}{\partial D} & =-\frac{F}{(C+1)\left(1+F^{2}\right)}<0 \\
\frac{\partial \mu^{P C}}{\partial D} & =-\frac{C}{(C+1)\left(1+F^{2}\right)}<0
\end{aligned}
$$


Proof of Proposition 5 The results and signs are evident upon partial differentiation of the interior solutions for $\mathrm{CO}$ in $\mathcal{S}_{\mu}^{C O}, x_{j}^{C O}, y_{j}^{C O}, j=1,2, \mu^{C O}$, in (18)-(22) with respect to $D$ :

$$
\begin{aligned}
\frac{\partial x_{1}^{C O}}{\partial D} & =\frac{1}{1+F^{2}}>0 \\
\frac{\partial x_{2}^{C O}}{\partial D} & =\frac{F}{1+F^{2}}>0 \\
\frac{\partial y_{1}^{C O}}{\partial D} & =-\frac{1}{(C+2)\left(1+F^{2}\right)}<0 \\
\frac{\partial y_{2}^{C O}}{\partial D} & =-\frac{F}{(C+2)\left(1+F^{2}\right)}<0 \\
\frac{\partial \mu^{C O}}{\partial D} & =-\frac{(2 C+3)}{(C+2)\left(1+F^{2}\right)}<0
\end{aligned}
$$

Proof of Proposition 6 The results and signs are evident upon partial differentiation of the interior solutions for $\mathrm{PC}$ in $\mathcal{S}^{P C}, x_{j}^{P C}, y_{j}^{P C}, j=1,2, \mu^{P C}$, in (13)-(17) with respect to $F$ while setting $T=0$ :

$$
\begin{aligned}
\frac{\partial x_{1}^{P C}}{\partial F} & =\frac{-\left(A_{2}-F A_{1}\right)+F\left(F A_{2}+A_{1}\right)-2 F D}{\left(1+F^{2}\right)^{2}} \\
\frac{\partial x_{2}^{P C}}{\partial F} & =\frac{-F\left(A_{2}-F A_{1}\right)-\left(F A_{2}+A_{1}\right)+\left(1-F^{2}\right) D}{\left(1+F^{2}\right)^{2}}<0 \\
\frac{\partial y_{1}^{P C}}{\partial F} & =\frac{\left(A_{2}-F A_{1}\right)-F\left(F A_{2}+A_{1}\right)+2 F D}{(C+1)\left(1+F^{2}\right)^{2}} \\
\frac{\partial y_{2}^{P C}}{\partial F} & =\frac{F\left(A_{2}-F A_{1}\right)+\left(F A_{2}+A_{1}\right)+\left(F^{2}-1\right) D}{(C+1)\left(1+F^{2}\right)^{2}}>0 \\
\frac{\partial \mu^{P C}}{\partial F} & =\frac{C\left[\left(A_{2}-F A_{1}\right)-F\left(F A_{2}+A_{1}\right)+2 F D\right]}{(C+1)\left(1+F^{2}\right)^{2}}
\end{aligned}
$$

The signs of (B-26), (B-28), and (B-30) are negative, positive, and positive, respectively, if $F<$ $\frac{\left(D-A_{1}\right)+\sqrt{A_{2}^{2}+\left(D-A_{1}\right)^{2}}}{A_{2}}$.

Proof of Proposition 7 The results are evident upon partial differentiation of the interior solutions for $\mathrm{CO}$ in $\mathcal{S}_{\mu}^{C O}, x_{j}^{C O}, y_{j}^{C O}, j=1,2, \mu^{C O}$, in (18)-(22) with respect to $F$ while setting 
$T=0$ :

$$
\begin{aligned}
\frac{\partial x_{1}^{C O}}{\partial F}= & \frac{\left[\left(2 F A_{1}-A_{2}\right)(C+1)\right]\left(1+F^{2}\right)}{(2 C+3)\left(1+F^{2}\right)^{2}} \\
& -\frac{2 F\left[F\left(F A_{1}-A_{2}\right)(C+1)+(2 C+3) D\right]}{(2 C+3)\left(1+F^{2}\right)^{2}}<0 \\
\frac{\partial x_{2}^{C O}}{\partial F}= & \frac{\left[-A_{1}(C+1)+(2 C+3) D\right]\left(1+F^{2}\right)}{(2 C+3)\left(1+F^{2}\right)^{2}} \\
& -\frac{2 F\left[\left(A_{2}-F A_{1}\right)(C+1)+(2 C+3) F D\right]}{(2 C+3)\left(1+F^{2}\right)^{2}}<0 \\
\frac{\partial y_{1}^{C O}}{\partial F}= & \frac{\left[2 F A_{1}(C+2)+A_{2}(C+1)\right]\left(1+F^{2}\right)}{(C+2)(2 C+3)\left(1+F^{2}\right)^{2}} \\
& -\frac{2 F\left[A_{1}\left(F^{2}(C+2)+2 C+3\right)+F A_{2}(C+1)-(2 C+3) D\right]}{(C+2)(2 C+3)\left(1+F^{2}\right)^{2}}>0 \\
\frac{\partial y_{2}^{C O}}{\partial F}= & \frac{\left[A_{1}(C+1)+2 F A_{2}(2 C+3)-(2 C+3) D\right]\left(1+F^{2}\right)}{(C+2)(2 C+3)\left(1+F^{2}\right)^{2}} \\
& -\frac{2 F\left[F A_{1}(C+1)+A_{2}\left(C+2+F^{2}(2 C+3)\right)-(2 C+3) F D\right]}{(C+2)(2 C+3)\left(1+F^{2}\right)^{2}}>0 \\
\frac{\partial \mu}{\partial F}= & \frac{\left[A_{2}(C+1)\right]\left(1+F^{2}\right)-2 F\left[A_{1}(C+1)+F A_{2}(C+1)-(2 C+3) D\right]}{(2 C+3)\left(1+F^{2}\right)^{2}}>0
\end{aligned}
$$

The signs for (B-32) and (B-34) follow because $-(C+1)\left[A_{1}+F A_{2}+F\left(A_{2}-F A_{1}\right)\right]-\left(F^{2}-\right.$ 1) $(2 C+3) D<0$. Likewise, the signs for (B-31), (B-33), and (B-35) follow because $(2 C+$ 3) $D>\left(A_{1}+F A_{2}\right)(C+1)$ (stemming from the negativity of $\mu^{C O}$ ), which implies $2 F(2 C+3) D>$ $2 F\left[\left(A_{1}+F A_{2}\right)(C+1)\right]>(C+1)\left[\left(F^{2}+1\right)\left(2 F A_{1}-A_{2}\right)-2 F^{2}\left(F A_{1}-A_{2}\right)\right]$.

\section{Appendix C. Closed-Loop Cournot Equilibrium}

When the RE producer's problem is dynamic, we solve it recursively to obtain a subgame perfect Cournot-Nash equilibrium, i.e., a closed-loop (CL) Cournot equilibrium. In effect, each producer acts as a Stackelberg leader in anticipating the impact of second-stage reactions when making firststage decisions. Starting in period 2, (5) implies $x_{2}=\left(D-x_{1}\right) / F$. Assuming an interior solution for $y_{2}$, KKT condition (10) implies:

$$
y_{2}=\frac{1}{(2+C)}\left(A_{2}-R T-\frac{\left(D-x_{1}\right)}{F}\right)
$$

Assuming an interior solution, the total production in period 2 is:

$$
x_{2}+y_{2}=\frac{1}{(2+C)}\left(A_{2}-R T+\frac{\left(D-x_{1}\right)}{F}(1+C)\right)
$$


Inserting these relationships into (1), we obtain

$$
\text { Maximise }_{x_{1} \geq 0} \quad x_{1}\left[A_{1}-\left(x_{1}+y_{1}\right)\right]+\frac{\left(D-x_{1}\right)}{F(2+C)}\left[\left(A_{2}-\frac{\left(D-x_{1}\right)}{F}\right)(1+C)+R T\right]
$$

Assuming an interior solution, we obtain $x_{1}^{C L}\left(y_{1}\right)$ as the reaction function from the first-order necessary condition of (C-3),

$$
x_{1}^{C L}\left(y_{1}\right)=\frac{\left(A_{1}-y_{1}\right) F^{2}(2+C)-F\left(A_{2}(1+C)+R T\right)+2 D(1+C)}{2\left(\left(1+F^{2}\right)(1+C)+F^{2}\right)}
$$

Using KKT condition (9) for the thermal producer, we obtain the closed-form solution for $x_{1}$ :

$$
x_{1}^{C L}=\frac{2 D(1+C)-F(1+C)\left(A_{2}-F A_{1}\right)+F(F-1) R T}{2(1+C)+F^{2}(3+2 C)}
$$

Inserting (C-5) in (4), (9), and (10), we obtain, respectively:

$$
\begin{gathered}
x_{2}^{C L}=\frac{F D(3+2 C)+(1+C)\left(A_{2}-F A_{1}\right)-(F-1) R T}{2(1+C)+F^{2}(3+2 C)} \\
y_{1}^{C L}=\frac{A_{1}\left(F^{2}(2+C)+2(1+C)\right)+F A_{2}(1+C)-2 D(1+C)-R T\left(2 F^{2}(2+C)+2(1+C)-F\right)}{(2+C)\left(2(1+C)+F^{2}(3+2 C)\right)} \\
y_{2}^{C L}=\frac{A_{2}\left(F^{2}(3+2 C)+1+C\right)+F A_{1}(1+C)-F D(3+2 C)-R T\left(\left(1+F^{2}\right)(3+2 C)-F\right)}{(2+C)\left(2(1+C)+F^{2}(3+2 C)\right)}
\end{gathered}
$$

Solving for $\mu$ in (3), we obtain:

$$
\mu^{C L}=\frac{2 A_{1}(1+C)^{2}+(1+C)(3+2 C)\left(F A_{2}-2 D\right)+R T(F(3+2 C)+2(1+C))}{(2+C)\left(2(1+C)+F^{2}(3+2 C)\right)}
$$

Through this CL Cournot model, we can assess the impact of new information available in a later stage, e.g., due to (possibly random) shocks to RE availability. As stated in Crampes and Moreaux (2001), “. . closed-loop equilibrium ... would result in a lower period 1 supply and a higher period 2 supply as compared with the open-loop equilibrium..." for the RE producer. The CL Cournot equilibrium takes into account the response of the thermal producer in period 2 due to any transfer of RE resource from period 1 to period 2, thereby putting more weight on period-2 production. Thus, the possibility of being able to "wait and see" in response to a shock to the RE stock, $D$, would have a similar effect. Towards that end, we observe that the main qualitative finding about the non-monotonic impact of storage efficiency, $F$, on the RE producer's profit still holds even when the producer has the possibility to anticipate second-stage decisions (see Figures C.26-C.29 and compare with Figures 19, 20, and 22 earlier). Hence, our implementation of a subgame perfect Nash equilibrium tackles the issue of whether the possibility of revising decisions over time changes the general conclusions (with the finding that it does not). 

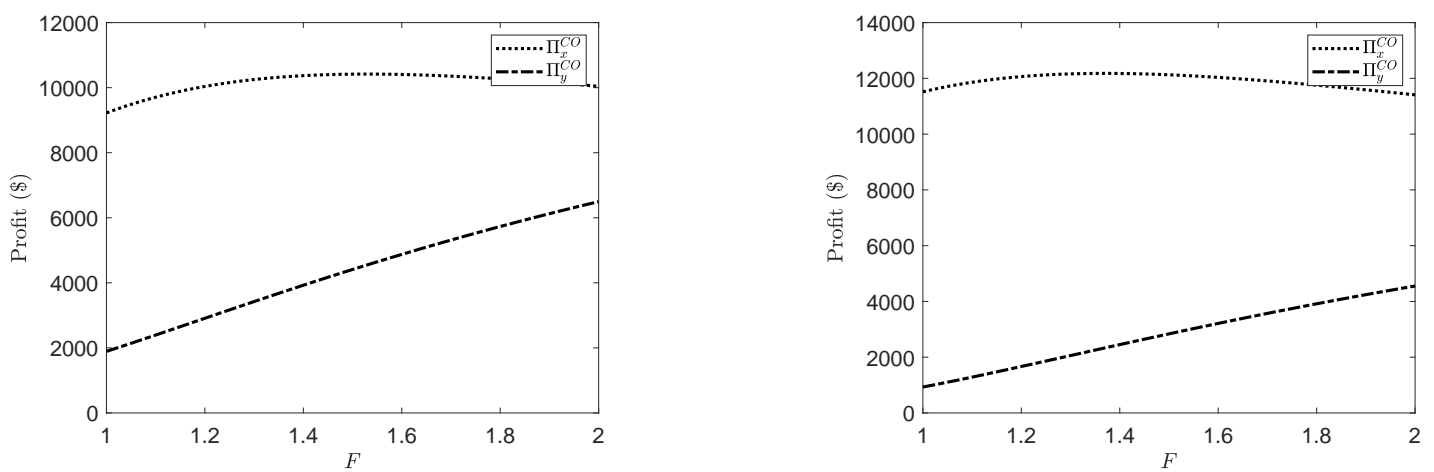

Figure C.26: Impact of RE storage efficiency on profit withFigure C.27: Impact of RE storage efficiency on profit with $\mathrm{CL}(T=\$ 0 / t)$

CL $(T=\$ 20 / t)$
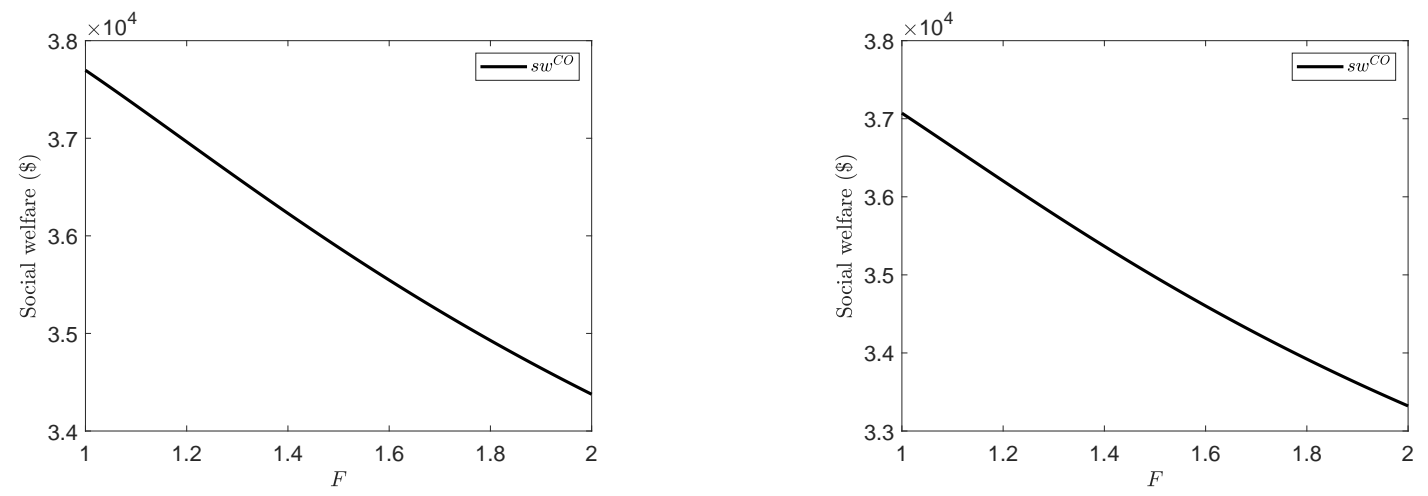

Figure C.28: Impact of RE storage efficiency on social wel-Figure C.29: Impact of RE storage efficiency on social welfare with CL $(T=\$ 0 / t)$ fare with CL $(T=\$ 20 / t)$

\section{Appendix D. PC and CO Equilibria with a Quadratic Emission Function}

The RE producer's problem is still (1)-(2) and leads to the same KKT conditions (5)-(7) or (3)-(5). Under a quadratic emission function, the thermal producer's problem is now:

$$
\text { Maximise }_{y_{j} \geq 0} \quad\left[A_{1}-\left(x_{1}+y_{1}\right)\right] y_{1}+\left[A_{2}-\left(x_{2}+y_{2}\right)\right] y_{2}-\frac{1}{2}(C+T R)\left(y_{1}^{2}+y_{2}^{2}\right)
$$

The KKT conditions under $\mathrm{CO}$ are:

$$
\begin{aligned}
& 0 \leq y_{1} \quad \perp-\left[A_{1}-\left(2 y_{1}+x_{1}\right)\right]+(C+T R) y_{1} \geq 0 \\
& 0 \leq y_{2} \quad \perp-\left[A_{2}-\left(2 y_{2}+x_{2}\right)\right]+(C+T R) y_{2} \geq 0
\end{aligned}
$$

Likewise, the KKT conditions under PC are:

$$
\begin{aligned}
& 0 \leq y_{1} \quad \perp-\left[A_{1}-\left(y_{1}+x_{1}\right)\right]+(C+T R) y_{1} \geq 0 \\
& 0 \leq y_{2} \quad \perp-\left[A_{2}-\left(y_{2}+x_{2}\right)\right]+(C+T R) y_{2} \geq 0
\end{aligned}
$$


Under PC, the interior solutions to (5)-(7) and (D-4)-(D-5) become:

$$
\begin{aligned}
x_{1}^{P C} & =\frac{F\left(F A_{1}-A_{2}\right)+D}{1+F^{2}} \\
x_{2}^{P C} & =\frac{A_{2}-F A_{1}+F D}{1+F^{2}} \\
y_{1}^{P C} & =\frac{A_{1}+F A_{2}-D}{\left(1+F^{2}\right)(1+C+R T)} \\
y_{2}^{P C} & =\frac{F\left(A_{1}+F A_{2}-D\right)}{\left(1+F^{2}\right)(1+C+T R)} \\
\mu^{P C} & =\frac{\left(A_{1}+F A_{2}-D\right)(C+T R)}{\left(1+F^{2}\right)(1+C+T R)}
\end{aligned}
$$

Likewise, the interior solutions to (3)-(5) and (D-2)-(D-3) under CO are:

$$
\begin{aligned}
x_{1}^{C O} & =\frac{F\left(F A_{1}-A_{2}\right)(1+C+T R)}{\left(1+F^{2}\right)(3+2(C+T R))}+\frac{D}{1+F^{2}} \\
x_{2}^{C O} & =\frac{\left(A_{2}-F A_{1}\right)(1+C+T R)}{\left(1+F^{2}\right)(3+2(C+T R))}+\frac{F D}{1+F^{2}} \\
y_{1}^{C O} & =\frac{\left[A_{1}\left(2+F^{2}\right)+F A_{2}-2 D\right](1+C+T R)+A_{1}\left(1+F^{2}\right)-D}{\left(1+F^{2}\right)(2+C+T R)(3+2(C+T R))} \\
y_{2}^{C O} & =\frac{\left[F A_{1}+A_{2}\left(2 F^{2}+1\right)-2 F D\right](1+C+T R)+A_{2}\left(1+F^{2}\right)-F D}{\left(1+F^{2}\right)(2+C+T R)(3+2(C+T R))} \\
\mu^{C O} & =\frac{\left(A_{1}+F A_{2}\right)(1+C+T R)-D(3+2(C+T R))}{\left(1+F^{2}\right)(2+C+T R)}
\end{aligned}
$$

Since the closed-form expressions are not easily amenable to analytical comparative statics, we investigate the robustness of the results in Propositions 1 and 3 numerically. In particular, we would like to know if a carbon tax under PC may actually increase peak thermal output and whether a carbon tax induces the RE producer to generate more during the off-peak period under PC than $\mathrm{CO}$ with a quadratic emission function. From Figures D.30-D.31, we cannot show that these results hold with a quadratic emission function for the given parameters (cf. Figures 2 and 3). This reversal of the previous results here with a quadratic emission function stems from the simple fact that the quadratic emission term effectively increases the value of the marginal cost, $C$, and removes the linear carbon tax, RT. Thus, Proposition 3 does not make sense in such a case.

By contrast as shown in Figures D.32-D.35, the counterintuitive results pertaining to RE storage efficiency in Proposition 6, $F$, still hold, viz., $F$ has a non-monotonic impact on the RE marginal value under PC even with a quadratic emission function (cf. Figure 13). As a result, there are "turning points" in the RE producer's off-peak production decisions (cf. Figure 16) and profit (cf. Figure 20). Hence, a conflict remains between private and public welfare due to the impact of $F$, even with a quadratic emission function as social welfare monotonically increases as RE storage efficiency increases (cf. Figure 22). 


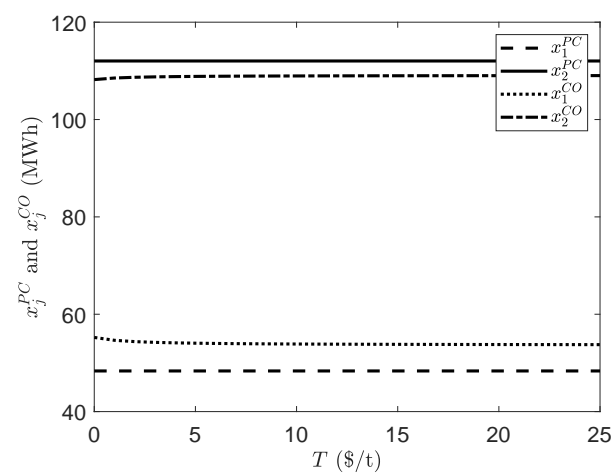

Figure D.30: Impact of carbon tax on RE production withFigure D.31: Impact of carbon tax on thermal production a quadratic emission function
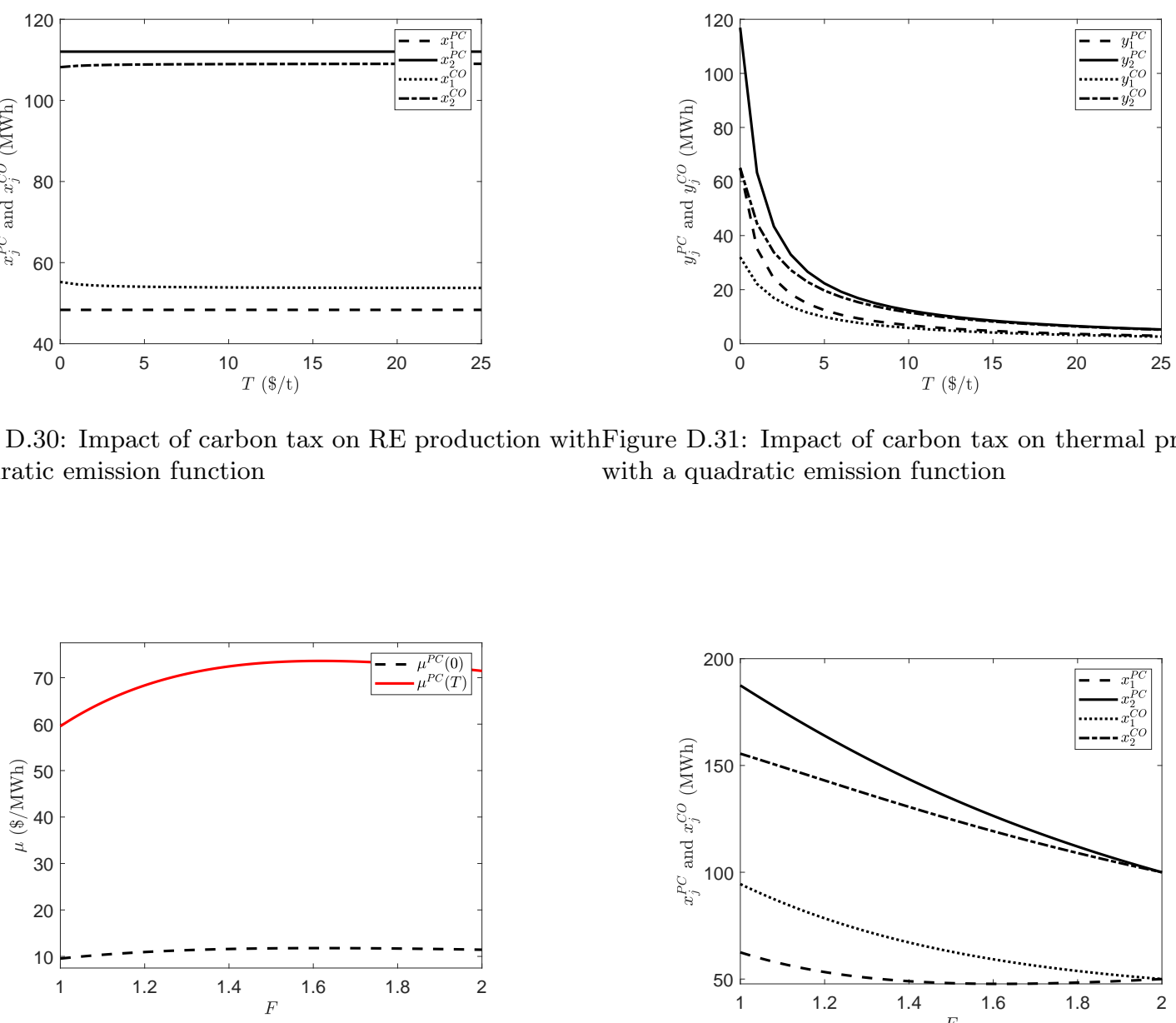

with a quadratic emission function

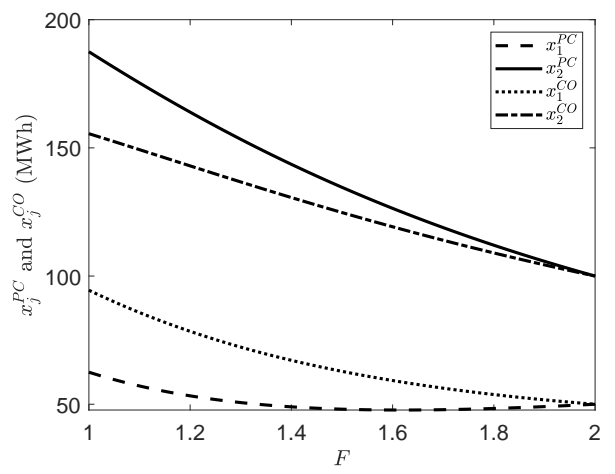

Figure D.32: Impact of RE storage efficiency on RE Figure D.33: Impact of RE storage efficiency on RE promarginal value under $\mathrm{PC}$ with a quadratic emission func- ${ }_{\text {duction with a quadratic emission function }(T=\$ 20 / t)}$
tion
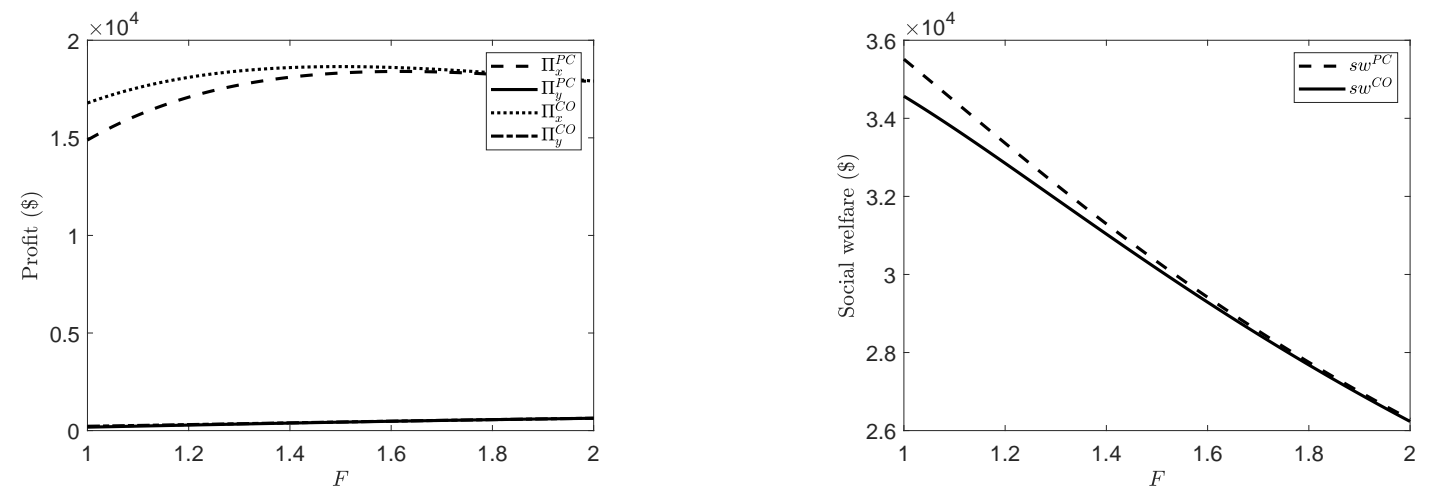

Figure D.34: Impact of RE storage efficiency on profit withFigure D.35: Impact of RE storage efficiency on social wela quadratic emission function $(T=\$ 20 / t)$

fare with a quadratic emission function $(T=\$ 20 / t)$ 